\title{
MATHEMATICAL MODEL OF HEAT TRANSFER THROUGH A CONDUCTIVE PIPE *
}

\author{
Matko Ljulj, Eduard Marušić-Paloka, Igor PaŽanin And Josip TAmbaČA**
}

\begin{abstract}
The standard engineer's model for heat transfer between the fluid flowing through the pipe and the exterior medium neglects the effects of the pipe's wall. The goal of this paper is to prove that they are not always negligible. Comparing the ratio between diffusivities of the fluid and the wall with the wall's thickness, using rigorous asymptotic analysis, we find five different models for effective description of the heat exchange process.
\end{abstract}

Mathematics Subject Classification. 35B40, 35Q79, 35J25.

Received August 11, 2020. Accepted February 5, 2021.

\section{INTRODUCTION}

A common situation encountered by engineers is heat transfer to fluid flowing through a tube. This can occur in heat exchangers, boilers, condensers, evaporators, radiators, and other process equipment. Therefore, it is useful to know how to model such situation. Most frequently the wall of the pipe is neglected as it is thin and highly conductive. However, we will show that this is not necessarily the case in all possible physical situations. We aim to derive the effective model by taking into account possible effects coming from the pipe's wall.

We study the heat conduction through the pipe filled with viscous fluid. There is a temperature difference between the fluid in the pipe and the exterior medium resulting with a heat exchange. There is a significant difference between the heat diffusivity of the wall of the pipe and the fluid, depending on both; the material the wall is made of and on the fluid the pipe is filled with. The pipe can be made of some metal (copper, steel, ...) that conducts the heat much better then the fluid, but it can also be made of PVC or concrete that conduct much weaker then the fluid. It can even have some isolating layer around it to prevent the heat exchange. To get some idea of physical coefficients we mention here that the thermal diffusivity (the coefficient appearing in the heat-conduction equation) is defined as

$$
\begin{aligned}
& \alpha=\frac{k}{\rho c_{p}}, k-\text { the heat conductivity, } \\
& \rho \text { - the density, } c_{p} \text { - the specific heat capacity. }
\end{aligned}
$$

Keywords and phrases. Asymptotic analysis, two-scale convergence, convection-diffusion equation, mathematical modeling, heat transfer.

* THIS PAPER IS DEVOTED TO THE MEMORY OF OUR FRIEND ANDRO MIKELIĆ.

Department of Mathematics, University of Zagreb, Bijenička Cesta 30, 10000 Zagreb, Croatia.

** Corresponding author: tambaca@math.hr 
The values of $\alpha$ (measured at room temperature in $\mathrm{m}^{2} / \mathrm{s}$ ) for different materials are given in the following table:

\begin{tabular}{ll}
\hline Material & Thermal diffusivity \\
\hline Copper & $\alpha=1.11 \times 10^{-4}$ \\
Steel & $\alpha=1.88 \times 10^{-5}$ \\
Aluminium & $\alpha=1.43 \times 10^{-7}$ \\
Glass & $\alpha=3.4 \times 10^{-7}$ \\
Rubber & $\alpha=0.8-1.3 \times 10^{-7}$ \\
PVC & $\alpha=8 \times 10^{-8}$ \\
Water & $\alpha=1.43 \times 10^{-7}$ \\
Alcohol & $\alpha=7 \times 10^{-8}$ \\
\hline
\end{tabular}

In view of that, the ratio between the thermal diffusivity of the pipe's wall and the liquid can be as high as $10^{4}$ or as low as $10^{-1}$.

The problem of heat exchange between the pipe and the exterior medium has been extensively studied in the literature. Asymptotic modeling in case of straight pipe can be found, for instance in [11,13], and the references therein. The effects of curvature for the process in curved pipe was studied in $[14,15]$. The effects of pipe dilatation, due to the heat, were considered in [16,17]. Similar results for heat conduction equation in a body containing a thin interlayer with high contrast conductivity was studied in [1], Chapter 9, Section 4. Like in our paper, the discrepancy in conductivity is described by $\varepsilon^{\gamma}$. Five different cases: very poorly conductive $(\gamma \geq 2)$, poorly conductive $(\gamma=1)$, conductive $(\gamma=0)$, highly conductive $(\gamma=-1)$ and very highly conductive interlayer $(\gamma \leq-2)$ were found. The study of a rod with three highly contrast coefficients was studied in Chapter 2 of [20]. Study of two linearly elastic materials made of material with different stiffnesses has been done in [2] and [8], using similar approach.

\section{THE PROBLEM}

After changing the variables by dividing with characteristic length $R$ (the radius of the pipe), the pipe and its wall are described (in cylindrical coordinates) as

$$
\begin{aligned}
P_{1}= & \left\{(r, \varphi, z) \in \mathbb{R}^{3} ; 0<\varphi<2 \pi, 0 \leq r<1,0<z<L\right\} \\
& - \text { the interior of the pipe, } \\
P_{\varepsilon}= & \left\{(r, \varphi, z) \in \mathbb{R}^{3} ; 0<\varphi<2 \pi, 1<r<1+\varepsilon, 0<z<L\right\} \\
& - \text { the wall of the pipe } \\
P_{1+\varepsilon}= & \left\{(r, \varphi, z) \in \mathbb{R}^{3} ; 0<\varphi<2 \pi, 0 \leq r<1+\varepsilon, 0<z<L\right\} .
\end{aligned}
$$

Using standard parametrization for the cylindrical coordinates $F(r, \varphi, z)=(r \cos \varphi, r \sin \varphi, z)$ we denote the physical sets in Cartesian coordinates

$$
\tilde{P}_{1}=F\left(P_{1}\right), \quad \tilde{P}_{\varepsilon}=F\left(P_{\varepsilon}\right), \quad \tilde{P}_{1+\varepsilon}=F\left(P_{1+\varepsilon}\right) .
$$

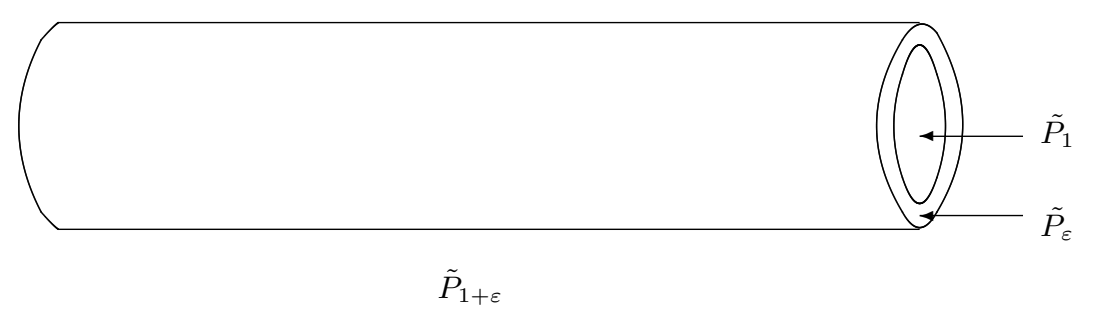


The equation of heat conduction that we are about to study is a simple stationary convection-diffusion equation. We denote by $\Theta^{\varepsilon}$ the temperature and by $w$ the Hagen-Poiseuille velocity through the pipe, i.e.

$$
w(r)=\frac{p_{\ell}-p_{r}}{4 \mu}\left(1-r^{2}\right),
$$

where $p_{\ell}$ and $p_{r}$ are the pressures on left and right ends of the pipe, respectively and $\mu$ is the dynamic viscosity. We assume that $p_{\ell}>p_{r}$.

$$
\begin{array}{ll}
\alpha_{f}\left(\frac{1}{r} \frac{\partial}{\partial r}\left(r \frac{\partial \Theta^{\varepsilon}}{\partial r}\right)+\frac{1}{r^{2}} \frac{\partial^{2} \Theta^{\varepsilon}}{\partial \varphi^{2}}+\frac{\partial^{2} \Theta^{\varepsilon}}{\partial z^{2}}\right)=R w(r) \frac{\partial \Theta^{\varepsilon}}{\partial z} & \text { in } P_{1}, \\
\alpha_{s}\left(\frac{1}{r} \frac{\partial}{\partial r}\left(r \frac{\partial \Theta^{\varepsilon}}{\partial r}\right)+\frac{1}{r^{2}} \frac{\partial^{2} \Theta^{\varepsilon}}{\partial \varphi^{2}}+\frac{\partial^{2} \Theta^{\varepsilon}}{\partial z^{2}}\right)=0 & \text { in } P_{\varepsilon}, \\
\alpha_{s} \frac{\partial \Theta^{\varepsilon}}{\partial r}=\zeta\left(g-\Theta^{\varepsilon}\right) & \text { for } r=1+\varepsilon, \\
\Theta^{\varepsilon}(1-, \varphi, z)=\Theta^{\varepsilon}(1+, \varphi, z)-\text { continuity of the temperature at } r=1, & \\
\alpha_{f} \frac{\partial \Theta^{\varepsilon}}{\partial r}(1-, \varphi, z)=\alpha_{s} \frac{\partial \Theta^{\varepsilon}}{\partial r}(1+, \varphi, z)-\text { continuity of the flux at } r=1, & \\
-\alpha_{f} \frac{\partial \Theta^{\varepsilon}}{\partial z}(r, \varphi, 0)+R w(r) \Theta^{\varepsilon}(r, \varphi, 0)=\lambda_{f}\left(F_{\ell}-\Theta^{\varepsilon}(r, \varphi, 0)\right), & 0<r<1, \\
\alpha_{f} \frac{\partial \Theta^{\varepsilon}}{\partial z}(r, \varphi, L)-R w(r) \Theta^{\varepsilon}(r, \varphi, L)=\lambda_{f}\left(F_{r}-\Theta^{\varepsilon}(r, \varphi, L)\right), & 0<r<1, \\
-\alpha_{s} \frac{\partial \Theta^{\varepsilon}}{\partial z}(r, \varphi, 0)=\lambda_{s}\left(F_{\ell}-\Theta^{\varepsilon}(r, \varphi, 0)\right) & \text { for } 1<r<1 \\
\alpha_{s} \frac{\partial \Theta^{\varepsilon}}{\partial z}(r, \varphi, L)=\lambda_{s}\left(F_{r}-\Theta^{\varepsilon}(r, \varphi, L)\right) & \text { for } 1<r<1
\end{array}
$$

Here $\alpha_{f}$ and $\alpha_{s}$ are the thermal diffusivities of the fluid and the solid, respectively. The heat exchange is described by the Robin boundary condition, with renormalized transfer coefficient on pipe's wall

$$
\zeta=\frac{h}{\rho c_{p}}
$$

where $h$ is the heat transfer coefficient, $c_{p}$ is the specific heat capacity and $\rho$ is the density, and $g$ is the exterior temperature. $\lambda_{f}$ and $\lambda_{s}, F_{\ell}$ and $F_{r}$ play the same role as $\zeta$ and $g$ on the ends of the pipe. The coefficient $h$ is proportional to the heat conductivity $k$ of the medium surrounding the pipe. That could be air or some other fluid. It also depends on the geometry of the pipe as well as on the convection around the pipe (if any) and it is usually computed from the Nusselt number of the surrounding medium. Different authors propose different ways to compute $h$. We mention here the law proposed by Churchill and Chu [3] (see also [5,7])

$$
h=\frac{k}{2 R}\left(0.6+\frac{0.387 \mathrm{Ra}^{1 / 6}}{\left(1+(0.669 / \mathrm{Pr})^{9 / 16}\right)^{8 / 27}}\right)^{2},
$$

where Ra and Pr are Rayleigh and Prandtl numbers, respectively.

As for the boundary conditions at the entry $z=0$ and at the exit $z=L$ of the pipe, for the sake of simplicity we have imposed the Robin boundary conditions. Another possibility, that can be treated similarly, would be to impose the Neumann condition. Imposing the Dirichlet condition on both ends has no physical sense. It would mean to impose the temperatures of the fluid entering the pipe and exiting the pipe while we are trying to model the process of cooling (or heating) the fluid in the pipe by surrounding medium. Bearing that in mind, it would make sense to impose the Dirichlet condition at the entry of the pipe, and either the Neumann or the Robin condition at the exit. 
For our analysis we prefer to write the equations in non-dimensional form. To do so we take the characteristic temperature $T_{c}$ and the characteristic velocity

$$
V=\int_{0}^{1} \int_{0}^{2 \pi} w(r) r \mathrm{~d} r \mathrm{~d} \varphi=\frac{\pi}{8 \mu}\left(p_{\ell}-p_{r}\right) \quad \text { (the Hagen-Poiseuille velocity). }
$$

Then we put

$$
\theta^{\varepsilon}=\frac{\Theta^{\varepsilon}}{T_{c}}, \quad v(r)=\frac{w(r)}{V}=\frac{2}{\pi}\left(1-r^{2}\right) .
$$

We define the Péclet number (for the fluid part, obviously, because in the solid part no convection appears)

$$
\mathrm{Pe}=\frac{L V}{\alpha_{f}}
$$

For the sake of our analysis we relate the ratio of two diffusivities $\alpha_{s} / \alpha_{f}$ with the relative thickness $\varepsilon$. More precisely, we assume that

$$
\frac{\alpha_{s}}{\alpha_{f}}=\kappa \varepsilon^{q},
$$

for some $q \in \mathbb{R}$. Since for most pipes the thickness is between 1 and $5 \mathrm{~mm}$, and the length is measured in meters, typical $\varepsilon$ is of order $10^{-3}$. It seems reasonable to relate the ratio of thermal diffusivities with that small parameter.

The goal of our paper is to perform the asymptotic analysis using the pipe's wall relative thickness $\varepsilon$ as the small parameter (relative, since $\varepsilon$ is the physical thickness of the pipe's wall divided by the radius $R$ ).

We also define the Nusselt numbers as

$$
N_{r}=\frac{R \zeta}{\alpha_{f}}, \quad N_{z}^{f}=\frac{\lambda_{f}}{\alpha_{f}}, \quad N_{z}^{s}=\frac{\lambda_{s}}{\alpha_{f}}
$$

and

$$
G=\frac{g}{T_{c}}
$$

Now our problem (2.1)-(2.7) reads

$$
\begin{aligned}
-\left(\frac{1}{r} \frac{\partial}{\partial r}\left(r \frac{\partial \theta^{\varepsilon}}{\partial r}\right)+\frac{1}{r^{2}} \frac{\partial^{2} \theta^{\varepsilon}}{\partial \varphi^{2}}+\frac{\partial^{2} \theta^{\varepsilon}}{\partial z^{2}}\right)+\operatorname{Pe} v(r) \frac{\partial \theta^{\varepsilon}}{\partial z} & =0 & & \text { in } P_{1}, \\
\frac{1}{r} \frac{\partial}{\partial r}\left(r \frac{\partial \theta^{\varepsilon}}{\partial r}\right)+\frac{1}{r^{2}} \frac{\partial^{2} \theta^{\varepsilon}}{\partial \varphi^{2}}+\frac{\partial^{2} \theta^{\varepsilon}}{\partial z^{2}} & =0 & & \text { in } P_{\varepsilon}, \\
\kappa \varepsilon^{q} \frac{\partial \theta^{\varepsilon}}{\partial r} & =N_{r}\left(G-\theta^{\varepsilon}\right) & & \text { for } r=1+\varepsilon, \\
\theta^{\varepsilon}(1-, \varphi, z) & =\theta^{\varepsilon}(1+, \varphi, z), & & \\
\frac{\partial \theta^{\varepsilon}}{\partial r}(1-, \varphi, z) & =\kappa \varepsilon^{q} \frac{\partial \theta^{\varepsilon}}{\partial r}(1+, \varphi, z), & & \\
-\frac{\partial \theta^{\varepsilon}}{\partial z}(r, \varphi, 0)+\operatorname{Pe} v \theta^{\varepsilon}(r, \varphi, 0) & =N_{z}^{f}\left(F_{\ell}-\theta^{\varepsilon}(r, \varphi, 0)\right) & & \text { for } 0<r<1, \\
\frac{\partial \theta^{\varepsilon}}{\partial z}(r, \varphi, L)-\operatorname{Pe} v \theta^{\varepsilon}(r, \varphi, L) & =N_{z}^{f}\left(F_{r}-\theta^{\varepsilon}(r, \varphi, L)\right) & & \text { for } 0<r<1, \\
-\kappa \varepsilon^{q} \frac{\partial \theta^{\varepsilon}}{\partial z}(r, \varphi, 0) & =N_{z}^{s}\left(F_{\ell}-\theta^{\varepsilon}(r, \varphi, 0)\right) & & \text { for } 1<r<1+\varepsilon, \\
\kappa \varepsilon^{q} \frac{\partial \theta^{\varepsilon}}{\partial z}(r, \varphi, L) & =N_{z}^{s}\left(F_{r}-\theta^{\varepsilon}(r, \varphi, L)\right) & & \text { for } 1<r<1+\varepsilon .
\end{aligned}
$$


The weak form is now formulated on a domain $P_{1+\varepsilon}=[0,1+\varepsilon) \times(0,2 \pi) \times(0, L)$ by: find $\theta^{\varepsilon} \in H^{1}\left(P_{1+\varepsilon}\right)$ such that

$$
\begin{aligned}
& \int_{0}^{1} \int_{0}^{2 \pi} \int_{0}^{L}\left(r \frac{\partial \theta^{\varepsilon}}{\partial r} \frac{\partial \psi}{\partial r}+\frac{1}{r} \frac{\partial \theta^{\varepsilon}}{\partial \varphi} \frac{\partial \psi}{\partial \varphi}+r \frac{\partial \theta^{\varepsilon}}{\partial z} \frac{\partial \psi}{\partial z}\right) \mathrm{d} z \mathrm{~d} \varphi \mathrm{d} r \\
& +\kappa \varepsilon^{q} \int_{1}^{1+\varepsilon} \int_{0}^{2 \pi} \int_{0}^{L}\left(r \frac{\partial \theta^{\varepsilon}}{\partial r} \frac{\partial \psi}{\partial r}+\frac{1}{r} \frac{\partial \theta^{\varepsilon}}{\partial \varphi} \frac{\partial \psi}{\partial \varphi}+r \frac{\partial \theta^{\varepsilon}}{\partial z} \frac{\partial \psi}{\partial z}\right) \mathrm{d} z \mathrm{~d} \varphi \mathrm{d} r \\
& \quad-\operatorname{Pe} \int_{0}^{1} \int_{0}^{2 \pi} \int_{0}^{L} v(r) \frac{\partial \psi}{\partial z} \theta^{\varepsilon} \mathrm{d} z \mathrm{~d} \varphi \mathrm{d} r \\
& +N_{r}(1+\varepsilon) \int_{0}^{2 \pi} \int_{0}^{L} \theta^{\varepsilon}(1+\varepsilon, \varphi, z) \psi(1+\varepsilon, \varphi, z) \mathrm{d} z \mathrm{~d} \varphi \\
& +\int_{0}^{1+\varepsilon} \int_{0}^{2 \pi} N_{z}(r) \theta^{\varepsilon}(r, \varphi, 0) \psi(r, \varphi, 0) r \mathrm{~d} r \mathrm{~d} \varphi \\
& +\int_{0}^{1+\varepsilon} \int_{0}^{2 \pi} N_{z}(r) \theta^{\varepsilon}(r, \varphi, L) \psi(r, \varphi, L) r \mathrm{~d} r \mathrm{~d} \varphi \\
& =N_{r}(1+\varepsilon) \int_{0}^{2 \pi} \int_{0}^{L} G(\varphi, z) \psi(1+\varepsilon, \varphi, z) \mathrm{d} z \mathrm{~d} \varphi \\
& +\int_{0}^{2 \pi} \int_{0}^{1+\varepsilon} N_{z}(r) F_{\ell}(r, \varphi) \psi(r, \varphi, 0) r \mathrm{~d} r \mathrm{~d} \varphi+\int_{0}^{2 \pi} \int_{0}^{1+\varepsilon} N_{z}(r) F_{r}(r, \varphi) \psi(r, \varphi, L) r \mathrm{~d} r \mathrm{~d} \varphi
\end{aligned}
$$

for any $\psi \in H^{1}\left(P_{1+\varepsilon}\right)$. Here we have used the notation

$$
N_{z}(r)= \begin{cases}N_{z}^{f} & \text { for } 0 \leq r<1 \\ N_{z}^{s} & \text { for } 1 \leq r \leq 1+\varepsilon\end{cases}
$$

for the piecewise constant function $N_{z}$. In addition we denote

$$
N_{z, \max }=\max _{r} N_{z}(r), \quad N_{z, \min }=\min _{r} N_{z}(r)>0 .
$$

\section{The MAIN RESUlT}

Before we start with rigorous analysis we want to announce the main result of the paper.

Depending on the ratio between the diffusivities of the fluid inside the pipe and of the pipe's wall, described by the power $q$ in (2.10), we obtain five effective models for describing the problem of heat exchange between the fluid flowing through the pipe and the exterior medium. In all cases the effective equation remains the same heat equation

$$
-\Delta \theta+\operatorname{Pe} v \frac{\partial \theta}{\partial z}=0 \text { in } \tilde{P}_{1}
$$

The difference lies in the effective boundary condition describing the heat exchange through the pipe's wall (parametrized in cylindrical coordinates by $S$ ). We have

- For $q<-1$ the thermal conductivity of the pipe's wall is so large so the temperature is constant in the entire wall of pipe. Only overall temperature $\int_{S} G$ influences the process. Furthermore, the effective boundary condition is the non-local Robin condition, that we believe to be new in the literature, of the form

$$
\theta(1, \varphi, z)=\text { const . on } S, \frac{\partial \bar{\theta}}{\partial r}(1)=N_{r}(\bar{G}-\bar{\theta}(1)),
$$


where

$$
\bar{\theta}(r)=\int_{0}^{2 \pi} \int_{0}^{L} \theta(r, \varphi, z) \mathrm{d} \varphi \mathrm{d} z, \bar{G}=\int_{0}^{2 \pi} \int_{0}^{L} G(\varphi, z) \mathrm{d} \varphi \mathrm{d} z
$$

are the mean values of the fluid temperature and the temperature of the exterior medium, respectively.

- For $q=-1$ the thermal conductivity of the pipe's wall is large enough that longitudinal propagation at the boundary is important. In fact, the two processes (in the fluid and in the pipe's wall) are of the same order and we get the coupled boundary value problem consisting of the heat conduction equation in the fluid and the curvilinear version of the heat equation (the Laplace-Beltrami operator replacing the Laplacean) in the 2D pipe's wall $S$. After decoupling, we get the effective wall law in the form of peculiar second order boundary condition

$$
\frac{\partial \theta}{\partial r}(1, \varphi, z)-\kappa \Delta_{S} \theta(1, \varphi, z)=N_{r}[G(\varphi, z)-\theta(1, \varphi, z)] \quad \text { on } S .
$$

So, due to the fact that the pipe's wall conducts very well, the heat is not only conducted across the pipe's wall but also along the pipe.

- For $-1<q<1$ no influence of the pipe's wall conductivity is present in the limit model (classical engineering case), i.e. the effective boundary condition is similar to the one we started with

$$
\frac{\partial \theta}{\partial r}(1, \varphi, z)=N_{r}[G(\varphi, z)-\theta(1, \varphi, z)] \quad \text { on } \quad S .
$$

- In case $q=1$ the thermal conductivity is small enough that it reduces the effective Nusselt number in the boundary condition from $N_{r}$ to

$$
\frac{\kappa N_{r}}{\kappa+N_{r}}=N_{r} \frac{\kappa}{\kappa+N_{r}}<N_{r} .
$$

We get the effective boundary condition of the form

$$
\frac{\partial \theta}{\partial r}(1, \varphi, z)=\frac{\kappa N_{r}}{\kappa+N_{r}}[G(\varphi, z)-\theta(1, \varphi, z)] \quad \text { on } \quad S .
$$

- The last case is when $q>1$. In that case the wall of the pipe conducts the temperature so badly that it effectively behaves as an insulator. The effective boundary condition is thus the homogeneous Neumann condition, as if there is no heat exchange between the pipe and the exterior medium

$$
\frac{\partial \theta}{\partial r}(1, \varphi, z)=0 \quad \text { on } \quad S .
$$

The original $\varepsilon$ problem is formulated on a joint fluid and thin wall domain. On the other hand all obtained effective models are given only on the fluid domain $\tilde{P}_{1}$ with different boundary conditions on the pipe's wall. Thus the effective models have simpler mathematical structure and can be solved more efficiently. However in a real life situations it may be difficult to decide which effective model to use. Thus in Section 7 we also formulate unified model (7.1) given on the fluid domain $\tilde{P}_{1}$ only which is $\varepsilon$ dependent and has the same asymptotics with respect to $\varepsilon$ as the original $\varepsilon$ problem (2.14). Furthermore to see the qualitatively different behavior of the solutions of the $\varepsilon$ model (2.14) in Section 8 we do numerical simulations in different regimes and compare their results with simulations for the limit models and the unified model (7.1).

\section{A priori estimates}

Before we start with the analysis let us introduce some notations. Additional sets in the cylindrical coordinates we will use are:

$$
S_{r}=\{r\} \times\langle 0,2 \pi\rangle \times\langle 0, L\rangle, \quad r \in\{1,1+\varepsilon, 2\}, \quad S=\langle 0,2 \pi\rangle \times\langle 0, L\rangle,
$$




$$
\begin{array}{ll}
\gamma_{1}^{\ell}=[0,1) \times(0,2 \pi) \times\{0\}, & \gamma_{1+\varepsilon}^{\ell}=[0,1+\varepsilon) \times(0,2 \pi) \times\{0\}, \\
\gamma_{1}^{r}=[0,1) \times(0,2 \pi) \times\{L\}, & \gamma_{1+\varepsilon}^{r}=[0,1+\varepsilon) \times(0,2 \pi) \times\{L\} .
\end{array}
$$

Using again standard parametrization for the cylindrical coordinates $F(r, \varphi, z)=(r \cos \varphi, r \sin \varphi, z)$ we also introduce the associated images under $F$ of the defined sets. We have already introduced $\tilde{P}_{1}=F\left(P_{1}\right), \tilde{P}_{1+\varepsilon}=$ $F\left(P_{1+\varepsilon}\right), \tilde{P}_{\varepsilon}=F\left(P_{\varepsilon}\right)$. In addition we define $\tilde{S}_{1}=F\left(S_{1}\right), \tilde{S}_{1+\varepsilon}=F\left(S_{1+\varepsilon}\right), \tilde{\gamma}_{1}^{r}=F\left(\gamma_{1}^{r}\right), \tilde{\gamma}_{1}^{\ell}=F\left(\gamma_{1}^{\ell}\right), \tilde{\gamma}_{1+\varepsilon}^{r}=$ $F\left(\gamma_{1+\varepsilon}^{r}\right), \tilde{\gamma}_{1+\varepsilon}^{\ell}=F\left(\gamma_{1+\varepsilon}^{\ell}\right)$. These sets correspond to the geometry in Cartesian coordinates. Furthermore we introduce the notation $\sim$ for functions defined in Cartesian coordinates, for instance for solution $\theta^{\varepsilon}$ we introduce the function $\tilde{\theta}^{\varepsilon}: \tilde{P}_{1+\varepsilon} \rightarrow \mathbb{R}$ such that

$$
\theta^{\varepsilon}=\tilde{\theta}^{\varepsilon} \circ F .
$$

Any function given in cylindrical coordinates is, obviously, assumed to be $2 \pi$-periodic in angular variable $\varphi$. To shorten the notation we will also use the following notation for integrals, for instance,

$$
\begin{aligned}
\int_{\tilde{P}_{1}} \tilde{f} \mathrm{~d} V & =\int_{P_{1}} f r \mathrm{~d} r \mathrm{~d} \varphi \mathrm{d} z=\int_{0}^{1} \int_{0}^{2 \pi} \int_{0}^{L} f r \mathrm{~d} r \mathrm{~d} \varphi \mathrm{d} z \\
\int_{\tilde{S}_{1+\varepsilon}} \tilde{f} \mathrm{~d} a & =\left.(1+\varepsilon) \int_{S} f\right|_{r=1+\varepsilon} \mathrm{d} \varphi \mathrm{d} z, \quad \int_{\tilde{\gamma}_{1+\varepsilon}^{\ell}} \tilde{f} \mathrm{~d} a=\int_{0}^{1+\varepsilon} \int_{0}^{2 \pi} f(r, \varphi, 0) r \mathrm{~d} r \mathrm{~d} \varphi .
\end{aligned}
$$

Before we start, let us rewrite the problem (2.14) in the weak formulation and in Cartesian coordinates: find $\tilde{\theta}^{\varepsilon} \in H^{1}\left(\tilde{P}_{1+\varepsilon}\right)$ such that

$$
\begin{aligned}
\int_{\tilde{P}_{1}} & \nabla \tilde{\theta}^{\varepsilon} \cdot \nabla \tilde{\psi} \mathrm{d} V+\kappa \varepsilon^{q} \int_{\tilde{P}_{\varepsilon}} \nabla \tilde{\theta}^{\varepsilon} \cdot \nabla \tilde{\psi} \mathrm{d} V-\operatorname{Pe} \int_{\tilde{P}_{1}} \tilde{v} \frac{\partial \tilde{\psi}}{\partial z} \tilde{\theta}^{\varepsilon} \mathrm{d} V \\
& +N_{r} \int_{\tilde{S}_{1+\varepsilon}} \tilde{\theta}^{\varepsilon} \tilde{\psi} \mathrm{d} a+\int_{\tilde{\gamma}_{1+\varepsilon}^{e}} N_{z} \tilde{\theta}^{\varepsilon} \tilde{\psi} \mathrm{d} a+\int_{\tilde{\gamma}_{1+\varepsilon}^{r}} N_{z} \tilde{\theta}^{\varepsilon} \tilde{\psi} \mathrm{d} a \\
= & N_{r} \int_{\tilde{S}_{1+\varepsilon}} \tilde{G} \tilde{\psi} \mathrm{d} a+\int_{\tilde{\gamma}_{1+\varepsilon}^{e}} N_{z} \tilde{F}_{\ell} \tilde{\psi} \mathrm{d} a+\int_{\tilde{\gamma}_{1+\varepsilon}^{r}} N_{z} \tilde{F}_{r} \tilde{\psi} \mathrm{d} a
\end{aligned}
$$

for all $\tilde{\psi} \in H^{1}\left(\tilde{P}_{1+\varepsilon}\right)$. Throughout this paper we assume that

$$
\tilde{G} \in L^{\infty}\left(\tilde{S}_{1+\varepsilon}\right), \quad \tilde{F}_{\ell} \in L^{\infty}\left(\tilde{\gamma}_{1+\varepsilon}^{\ell}\right), \quad \tilde{F}_{r} \in L^{\infty}\left(\tilde{\gamma}_{1+\varepsilon}^{r}\right) .
$$

Under that assumption, the existence of weak solution can be found, for example, in [4].

Theorem 4.1. Let $\tilde{\theta}^{\varepsilon} \in H^{1}\left(\tilde{P}_{1+\varepsilon}\right)$ be a solution to the problem (2.11)-(2.13), under the assumption (4.2). Suppose that

$$
\mathrm{Pe}<\frac{3 \pi N_{z}^{f}}{8}
$$

Then

(a) there exists a constant

$$
C=C\left(N_{r}, N_{z},|G|_{L^{2}\left(\tilde{S}_{1+\varepsilon}\right)},\left|F_{r}\right|_{L^{2}\left(\tilde{\gamma}_{1+\varepsilon}^{r}\right)},\left|F_{\ell}\right|_{L^{2}\left(\tilde{\gamma}_{1+\varepsilon}^{\ell}\right)}\right)>0,
$$

independent on $\varepsilon$, such that

$$
\frac{1}{2}\left|\nabla \tilde{\theta}^{\varepsilon}\right|_{L^{2}\left(\tilde{P}_{1}\right)}^{2}+\kappa \varepsilon^{q}\left|\nabla \tilde{\theta}^{\varepsilon}\right|_{L^{2}\left(\tilde{P}_{\varepsilon}\right)}^{2}+N_{r}\left|\tilde{\theta}^{\varepsilon}\right|_{L^{2}\left(\tilde{S}_{1+\varepsilon}\right)}^{2}+\left|\sqrt{N_{z}} \tilde{\theta}^{\varepsilon}\right|_{L^{2}\left(\tilde{\gamma}_{1+\varepsilon}^{r}\right)}^{2}+\left|\sqrt{N_{z}} \tilde{\theta}^{\varepsilon}\right|_{L^{2}\left(\tilde{\gamma}_{1+\varepsilon}^{e}\right)}^{2} \leq C .
$$


(b) $\tilde{\theta}^{\varepsilon} \in L^{\infty}\left(\tilde{P}_{1+\varepsilon}\right)$ and there exists a constant

$$
M=M\left(N_{r}, N_{z},|G|_{L^{\infty}\left(\tilde{S}_{1+\varepsilon}\right)},\left|F_{r}\right|_{L^{\infty}\left(\tilde{\gamma}_{1+\varepsilon}^{r}\right)},\left|F_{\ell}\right|_{L^{\infty}\left(\tilde{\gamma}_{1+\varepsilon}^{\ell}\right)}\right)>0,
$$

independent on $\varepsilon$, such that

$$
\left|\tilde{\theta}^{\varepsilon}\right|_{L^{\infty}\left(\tilde{P}_{1+\varepsilon}\right)} \leq M
$$

For the proof we use the idea of Stampacchia [21] (see also [18]). Thus we first state classical Stampacchia's lemma.

Lemma 4.2. Let $\Omega \subset \mathbb{R}^{n}$ be a bounded domain and $\mathcal{G}: \mathbb{R} \rightarrow \mathbb{R}$ be a Lipschitz function such that $\mathcal{G}(0)=0$. Then, for any $u \in W^{1, p}(\Omega), 1<p<\infty$, we have $\mathcal{G}(u) \in W^{1, p}(\Omega)$ and

$$
\nabla \mathcal{G}(u)=\mathcal{G}^{\prime}(u) \nabla u .
$$

To continue we need a technical results:

Lemma 4.3. For any $\varepsilon \in\langle 0,1\rangle$ and $\tilde{\psi} \in H^{1}\left(\tilde{P}_{1+\varepsilon}\right)$ the following inequality holds

$$
|\tilde{\psi}|_{L^{2}\left(\tilde{P}_{1+\varepsilon}\right)}^{2} \leq 2\left(\left|\frac{\partial \tilde{\psi}}{\partial r}\right|_{L^{2}\left(\tilde{P}_{1+\varepsilon}\right)}^{2}+|\tilde{\psi}|_{L^{2}\left(\tilde{S}_{1+\varepsilon}\right)}^{2}\right) .
$$

Proof. Let $\psi$ be a smooth function. Then

$$
\begin{aligned}
|\psi(r, \varphi, z)| & =\left|-\int_{r}^{1+\varepsilon} \frac{\partial \psi}{\partial r}(\rho, \varphi, z) \mathrm{d} \rho+\psi(1+\varepsilon, \varphi, z)\right| \\
& \leq\left(\int_{r}^{1+\varepsilon}\left(\frac{\partial \psi}{\partial r}(\rho, \varphi, z)\right)^{2} \rho \mathrm{d} \rho\right)^{\frac{1}{2}}\left(\int_{r}^{1+\varepsilon} \frac{\mathrm{d} \rho}{\rho}\right)^{\frac{1}{2}}+|\psi(1+\varepsilon, \varphi, z)| .
\end{aligned}
$$

We take the square, estimate, multiply by $r$ and integrate over $P_{1+\varepsilon}$ to get

$$
\begin{aligned}
\int_{P_{1+\varepsilon}}|\psi(\rho, \varphi, z)|^{2} \rho \mathrm{d} \rho \mathrm{d} \varphi \mathrm{d} z \leq & 2 \int_{P_{1+\varepsilon}}\left(\frac{\partial \psi}{\partial r}(\rho, \varphi, z)\right)^{2} \rho \mathrm{d} \rho \mathrm{d} \varphi \mathrm{d} z \\
& \times \int_{0}^{1+\varepsilon}(\ln (1+\varepsilon)-\ln r) r \mathrm{~d} r+(1+\varepsilon)^{2} \int_{S_{1+\varepsilon}}|\psi|^{2} \\
= & \frac{1}{2}(1+\varepsilon)^{2} \int_{\tilde{P}_{1+\varepsilon}}\left(\frac{\partial \tilde{\psi}}{\partial r}\right)^{2}+2 \int_{\tilde{S}_{1+\varepsilon}}|\tilde{\psi}|^{2}
\end{aligned}
$$

since $\int_{\tilde{S}_{1+\varepsilon}}=(1+\varepsilon) \int_{S_{1+\varepsilon}}$.

Proof of Theorem 4.1. First of all, due to the Theorem 3.14. from [19] we know that for any solution of (2.11)(2.13) we have $\tilde{\theta}^{\varepsilon} \in C^{0, \gamma}\left(\tilde{P}_{1+\varepsilon}\right)$, for some $\gamma>0$. Furthermore, there exists some $C>0$ such that

$$
\begin{aligned}
\left|\tilde{\theta}^{\varepsilon}\right|_{C^{0, \gamma}\left(\tilde{P}_{1+\varepsilon}\right)} & =\left|\tilde{\theta}^{\varepsilon}\right|_{L^{\infty}\left(\tilde{P}_{1+\varepsilon}\right)}+\sup _{x \neq y} \frac{\left|\tilde{\theta}^{\varepsilon}(x)-\tilde{\theta}^{\varepsilon}(y)\right|}{|x-y|} \\
& \leq C\left(\left|\tilde{\theta}^{\varepsilon}\right|_{L^{2}\left(\tilde{P}_{1+\varepsilon}\right)}+|G|_{L^{\infty}\left(\tilde{S}_{1+\varepsilon}\right)}+\left|F_{r}\right|_{L^{\infty}\left(\tilde{\gamma}_{1+\varepsilon}^{r}\right)}+\left|F_{\ell}\right|_{L^{\infty}\left(\tilde{\gamma}_{1+\varepsilon}^{e}\right)}\right) .
\end{aligned}
$$


However, as far as we know, the constant $C$ depends on $\varepsilon$ and we have no idea how. Thus, we can use the regularity of $\tilde{\theta}^{\varepsilon}$, but the estimate is of no use to us.

We start the proof of the estimates (4.4) and (4.5). Let

$$
\theta_{+}^{\varepsilon}(\mathbf{x})=\max \left\{0, \tilde{\theta}^{\varepsilon}(\mathbf{x})\right\}, \theta_{-}^{\varepsilon}(\mathbf{x})=\max \left\{0,-\tilde{\theta}^{\varepsilon}(\mathbf{x})\right\} .
$$

Notice that the functions $\mathcal{G}(t)=\max \{0, t\}$ and $\mathcal{H}(t)=\max \{0,-t\}$ are Lipschitz and $\mathcal{G}(0)=\mathcal{H}(0)=0$, so that Lemma 4.2 applies. Furthermore

$$
\mathcal{G}^{\prime}(t)=\left\{\begin{array}{ll}
1, & t>0 \\
0, & t<0
\end{array}, \quad \mathcal{H}^{\prime}(t)=\left\{\begin{array}{ll}
-1, & t<0 \\
0, & t>0
\end{array},\right.\right.
$$

so that

$$
\nabla \theta_{+}^{\varepsilon}(\mathbf{x})=\left\{\begin{array}{ll}
\nabla \tilde{\theta}^{\varepsilon}(\mathbf{x}) & \text { if } \mathbf{x} \text { is such that } \tilde{\theta}^{\varepsilon}(\mathbf{x})>0 \\
0 & \text { if } \mathbf{x} \text { is such that } \tilde{\theta}^{\varepsilon}(\mathbf{x})<0
\end{array},\right.
$$

and

$$
\nabla \theta_{-}^{\varepsilon}(\mathbf{x})= \begin{cases}-\nabla \tilde{\theta}^{\varepsilon}(\mathbf{x}) & \text { if } \mathbf{x} \text { is such that } \tilde{\theta}^{\varepsilon}(\mathbf{x})<0 \\ 0 & \text { if } \mathbf{x} \text { is such that } \tilde{\theta}^{\varepsilon}(\mathbf{x})>0\end{cases}
$$

Obviously $\theta_{+}^{\varepsilon}, \theta_{-}^{\varepsilon} \geq 0$ and $\tilde{\theta}^{\varepsilon}=\theta_{+}^{\varepsilon}-\theta_{-}^{\varepsilon}$, while $\left|\tilde{\theta}^{\varepsilon}\right|=\theta_{+}^{\varepsilon}+\theta_{-}^{\varepsilon}$. We derive the estimate for $\theta_{+}^{\varepsilon}$. The estimate for $\theta_{-}^{\varepsilon}$ can be derived analogously. From two estimates for positive and negative part one gets directly the estimate for $\tilde{\theta}^{\varepsilon}$.

For $\lambda \geq 0$ we test the equations (2.11) and (2.12) by $\left(\theta_{+}^{\varepsilon}\right)^{\lambda+1}$ (however in Cartesian coordinates). That is a suitable test function since we know that $\tilde{\theta}^{\varepsilon} \in H^{1}\left(\tilde{P}_{1+\varepsilon}\right) \cap L^{\infty}\left(\tilde{P}_{1+\varepsilon}\right)$. We get

$$
\begin{aligned}
(\lambda+ & 1)\left(\int_{\tilde{P}_{1}}\left|\nabla \theta_{+}^{\varepsilon}\right|^{2}\left(\theta_{+}^{\varepsilon}\right)^{\lambda}+\kappa \varepsilon^{q} \int_{\tilde{P}_{\varepsilon}}\left|\nabla \theta_{+}^{\varepsilon}\right|^{2}\left(\theta_{+}^{\varepsilon}\right)^{\lambda}\right)+N_{r} \int_{\tilde{S}_{1+\varepsilon}}\left(\theta_{+}^{\varepsilon}\right)^{\lambda+2} \\
& +\int_{\tilde{\gamma}_{1+\varepsilon}^{r}} N_{z}\left(\theta_{+}^{\varepsilon}\right)^{\lambda+2}+\int_{\tilde{\gamma}_{1+\varepsilon}^{\ell}} N_{z}\left(\theta_{+}^{\varepsilon}\right)^{\lambda+2}-(\lambda+1) \operatorname{Pe} \int_{\tilde{P}_{1}} v\left(\theta_{+}^{\varepsilon}\right)^{\lambda+1} \frac{\partial \theta_{+}^{\varepsilon}}{\partial z} \\
= & N_{r} \int_{\tilde{S}_{1+\varepsilon}} G\left(\theta_{+}^{\varepsilon}\right)^{\lambda+1}+\int_{\tilde{\gamma}_{1+\varepsilon}^{r}} N_{z} F_{r}^{\varepsilon}\left(\theta_{+}^{\varepsilon}\right)^{\lambda+1}+\int_{\tilde{\gamma}_{1+\varepsilon}^{\ell}} N_{z} F_{\ell}^{\varepsilon}\left(\theta_{+}^{\varepsilon}\right)^{\lambda+1}
\end{aligned}
$$

As for the left-hand side, we obviously have

$$
\left|\nabla \theta_{+}^{\varepsilon}\right|^{2}\left(\theta_{+}^{\varepsilon}\right)^{\lambda}=\frac{1}{\left(1+\frac{\lambda}{2}\right)^{2}}\left|\nabla\left[\left(\theta_{+}^{\varepsilon}\right)^{1+\frac{\lambda}{2}}\right]\right|^{2}
$$

so that

$$
\begin{aligned}
& (\lambda+1)\left(\int_{\tilde{P}_{1}}\left|\nabla \theta_{+}^{\varepsilon}\right|^{2}\left(\theta_{+}^{\varepsilon}\right)^{\lambda}+\kappa \varepsilon^{q} \int_{\tilde{P}_{\varepsilon}}\left|\nabla \theta_{+}^{\varepsilon}\right|^{2}\left(\theta_{+}^{\varepsilon}\right)^{\lambda}\right) \\
& =\frac{1+\lambda}{\left(1+\frac{\lambda}{2}\right)^{2}}\left(\int_{\tilde{P}_{1}}\left|\nabla\left[\left(\theta_{+}^{\varepsilon}\right)^{1+\frac{\lambda}{2}}\right]\right|^{2}+\kappa \varepsilon^{q} \int_{\tilde{P}_{\varepsilon}}\left|\nabla\left[\left(\theta_{+}^{\varepsilon}\right)^{1+\frac{\lambda}{2}}\right]\right|^{2}\right) .
\end{aligned}
$$

Furthermore

$$
\begin{aligned}
-(\lambda+1) \int_{\tilde{P}_{1}} v\left(\theta_{+}^{\varepsilon}\right)^{\lambda+1} \frac{\partial \theta_{+}^{\varepsilon}}{\partial z} & =-\frac{\lambda+1}{\lambda+2} \int_{\tilde{P}_{1}} v \frac{\partial}{\partial z}\left[\left(\theta_{+}^{\varepsilon}\right)^{\lambda+2}\right] \\
& =\frac{\lambda+1}{2+\lambda}\left(\int_{\tilde{\gamma}_{1}^{e}} v\left(\theta_{+}^{\varepsilon}\right)^{\lambda+2}-\int_{\tilde{\gamma}_{1}^{r}} v\left(\theta_{+}^{\varepsilon}\right)^{\lambda+2}\right) .
\end{aligned}
$$


The first term in the right hand side of (4.9) is positive and the second one, since $v=\frac{2}{\pi}\left(1-r^{2}\right)$, can be estimated as

$$
\int_{\tilde{\gamma}_{1}^{r}} v\left(\theta_{+}^{\varepsilon}\right)^{\lambda+2} \leq \frac{2}{\pi} \int_{\tilde{\gamma}_{1}^{r}}\left|\left(\theta_{+}^{\varepsilon}\right)^{1+\frac{\lambda}{2}}\right|^{2} .
$$

The Young inequality with $r=\frac{2+\lambda}{1+\lambda}, r^{\prime}=2+\lambda$, as before, gives

$$
\begin{aligned}
\int_{\tilde{S}_{1+\varepsilon}} G\left(\theta_{+}^{\varepsilon}\right)^{\lambda+1} & =\int_{\tilde{S}_{1+\varepsilon}} \frac{1}{\tau} G \tau\left(\theta_{+}^{\varepsilon}\right)^{\lambda+1} \\
& \leq \frac{1}{\lambda+2}\left(\frac{1}{\tau}|G|_{L^{2+\lambda}\left(\tilde{S}_{1+\varepsilon}\right)}\right)^{2+\lambda}+\frac{1+\lambda}{2+\lambda} \tau^{\frac{2+\lambda}{1+\lambda}}\left|\theta_{+}^{\varepsilon}\right|_{L^{2+\lambda}\left(\tilde{S}_{1+\varepsilon}\right)}^{\lambda+2}
\end{aligned}
$$

For

$$
\tau=\left(\frac{1}{4} \frac{2+\lambda}{1+\lambda}\right)^{\frac{1+\lambda}{2+\lambda}}
$$

we get

$$
\frac{1+\lambda}{2+\lambda} \tau^{\frac{2+\lambda}{1+\lambda}}=\frac{1}{4}, \quad \frac{1}{\lambda+2}\left(\frac{1}{\tau}\right)^{2+\lambda}=\left(4 \frac{\lambda+1}{\lambda+2}\right)^{1+\lambda} \frac{1}{\lambda+2}<\frac{4^{1+\lambda}}{\lambda+2}
$$

and therefore

$$
\begin{aligned}
N_{r} \int_{\tilde{S}_{1+\varepsilon}} G\left(\theta_{+}^{\varepsilon}\right)^{\lambda+1} \leq & \frac{1}{\lambda+2} \frac{N_{r}}{4}\left(4|G|_{L^{2+\lambda}\left(\tilde{S}_{1+\varepsilon}\right)}\right)^{2+\lambda} \\
& +\frac{N_{r}}{4}\left|\left(\theta_{+}^{\varepsilon}\right)^{1+\frac{\lambda}{2}}\right|_{L^{2}\left(\tilde{S}_{1+\varepsilon}\right)}^{2}
\end{aligned}
$$

Using the same idea we prove that for $i \in\{r, \ell\}$

$$
\begin{aligned}
\int_{\tilde{\gamma}_{1+\varepsilon}^{i}} N_{z} F_{i}\left(\theta_{+}^{\varepsilon}\right)^{\lambda+1} \leq & \frac{1}{4(\lambda+2)}\left(4\left|\sqrt[2+\lambda]{N_{z}} F_{i}\right|_{L^{2+\lambda}\left(\tilde{\gamma}_{1+\varepsilon}^{i}\right)}\right)^{2+\lambda} \\
& +\frac{1}{4}\left|\sqrt{N_{z}}\left(\theta_{+}^{\varepsilon}\right)^{1+\frac{\lambda}{2}}\right|_{L^{2}\left(\tilde{\gamma}_{1+\varepsilon}^{i}\right)}^{2}
\end{aligned}
$$

At this point we need the assumption (4.11), i.e. that

$$
\mathrm{Pe}<\frac{3 \pi N_{z}^{f}}{8} \frac{2+\lambda}{1+\lambda}
$$

since then there are no negative terms coming from the left hand side of (4.7). Combining (4.7)-(4.10) we arrive at

$$
\begin{aligned}
& \frac{(1+\lambda)}{2\left(1+\frac{\lambda}{2}\right)^{2}}\left(\int_{\tilde{P}_{1}}\left|\nabla\left[\left(\theta_{+}^{\varepsilon}\right)^{1+\frac{\lambda}{2}}\right]\right|^{2}+\kappa \varepsilon^{q} \int_{\tilde{P}_{\varepsilon}}\left|\nabla\left[\left(\theta_{+}^{\varepsilon}\right)^{1+\frac{\lambda}{2}}\right]\right|^{2}\right) \\
& \quad+\frac{N_{r}}{2} \int_{\tilde{S}_{1+\varepsilon}}\left|\left(\theta_{+}^{\varepsilon}\right)^{1+\frac{\lambda}{2}}\right|^{2}+\frac{1}{2} \int_{\tilde{\gamma}_{1+\varepsilon}^{\ell}} N_{z}\left|\left(\theta_{+}^{\varepsilon}\right)^{1+\frac{\lambda}{2}}\right|^{2} \\
& \leq \frac{4^{1+\lambda}}{\lambda+2}\left[\left(\left|\sqrt[2+\lambda]{N_{z}} F_{r}\right|_{L^{2+\lambda}\left(\tilde{\gamma}_{1+\varepsilon}^{r}\right)}\right)^{2+\lambda}+\left(\left|\sqrt[2+\lambda]{N_{z}} F_{\ell}\right|_{L^{2+\lambda}\left(\tilde{\gamma}_{1+\varepsilon}^{\ell}\right)}\right)^{2+\lambda}\right] \\
& \quad+\frac{1}{\lambda+2} \frac{N_{r}}{4}\left(4|G|_{L^{2+\lambda}\left(\tilde{S}_{1+\varepsilon}\right)}\right)^{2+\lambda} .
\end{aligned}
$$


Obviously, for $\lambda=0$, under the assumption that Pe is such that (4.11) holds, we get the standard variational estimate

$$
\begin{gathered}
\int_{\tilde{P}_{1}}\left|\nabla \theta_{+}^{\varepsilon}\right|^{2}+\kappa \varepsilon^{q} \int_{\tilde{P}_{\varepsilon}}\left|\nabla \theta_{+}^{\varepsilon}\right|^{2}+N_{r} \int_{\tilde{S}_{1+\varepsilon}}\left|\theta_{+}^{\varepsilon}\right|^{2}+\int_{\tilde{\gamma}_{1+\varepsilon}^{\ell}} N_{z}\left|\theta_{+}^{\varepsilon}\right|^{2} \\
\leq 4\left|\sqrt{N_{z}} F_{r}\right|_{L^{2}\left(\tilde{\gamma}_{1+\varepsilon}^{r}\right)}^{2}+4\left|\sqrt{N_{z}} F_{\ell}\right|_{L^{2}\left(\tilde{\gamma}_{1+\varepsilon}^{\ell}\right)}^{2}+4 N_{r}|G|_{L^{2}\left(\tilde{S}_{1+\varepsilon}\right)}^{2} .
\end{gathered}
$$

Next step is to take the limit as $\lambda \rightarrow+\infty$. First we can assume that $\lambda$ is large enough such that (4.11) holds. We can do that, since we have imposed the condition (4.3) and $\lim _{\lambda \rightarrow \infty} \frac{\lambda+2}{\lambda+1}=1$. Next, we suppose that it is large enough to have

$$
\lambda \geq \frac{4}{N_{r}}-2
$$

thus $N_{r} \geq 4 /(\lambda+2)$, so that

$$
\frac{(1+\lambda)}{2\left(1+\frac{\lambda}{2}\right)^{2}} \leq \frac{N_{r}}{2}
$$

and

$$
\begin{aligned}
& \frac{(1+\lambda)}{2\left(1+\frac{\lambda}{2}\right)^{2}}\left(\int_{\tilde{P}_{1}}\left|\nabla\left[\left(\theta_{+}^{\varepsilon}\right)^{1+\frac{\lambda}{2}}\right]\right|^{2}+\kappa \varepsilon^{q} \int_{\tilde{P}_{\varepsilon}}\left|\nabla\left[\left(\theta_{+}^{\varepsilon}\right)^{1+\frac{\lambda}{2}}\right]\right|^{2}+\int_{\tilde{S}_{1+\varepsilon}}\left|\left(\theta_{+}^{\varepsilon}\right)^{1+\frac{\lambda}{2}}\right|^{2}\right) \\
& \leq \frac{4^{1+\lambda}}{\lambda+2}\left[\left(\left|\sqrt[2+\lambda]{N_{z}} F_{r}\right|_{L^{2+\lambda}\left(\tilde{\gamma}_{1+\varepsilon}^{r}\right)}\right)^{2+\lambda}+\left(\left|\sqrt[2+\lambda]{N_{z}} F_{\ell}\right|_{L^{2+\lambda}\left(\tilde{\gamma}_{1+\varepsilon}^{\ell}\right)}\right)^{2+\lambda}\right] \\
& \quad+\frac{1}{\lambda+2} \frac{N_{r}}{4}\left(4|G|_{L^{2+\lambda}\left(\tilde{S}_{1+\varepsilon}\right)}\right)^{2+\lambda} .
\end{aligned}
$$

We have two cases: $q>0$ and $q \leq 0$.

For $q>0$ and $\varepsilon$ small enough such that $\kappa \varepsilon^{q} \leq 1$, we have

$$
\begin{aligned}
& \frac{(1+\lambda) \kappa \varepsilon^{q}}{2\left(1+\frac{\lambda}{2}\right)^{2}}\left(\int_{P_{1+\varepsilon}}\left|\nabla\left[\left(\theta_{+}^{\varepsilon}\right)^{1+\frac{\lambda}{2}}\right]\right|^{2}+\int_{\tilde{S}_{1+\varepsilon}}\left|\left(\theta_{+}^{\varepsilon}\right)^{1+\frac{\lambda}{2}}\right|^{2}\right) \\
& \quad \leq \frac{4^{1+\lambda}}{\lambda+2}\left[\left(\left|\sqrt[2+\lambda]{N_{z}} F_{r}\right|_{L^{2+\lambda}\left(\tilde{\gamma}_{1+\varepsilon}^{r}\right)}\right)^{2+\lambda}+\left(\left|\sqrt[2+\lambda]{N_{z}} F_{\ell}\right|_{L^{2+\lambda}\left(\tilde{\gamma}_{1+\varepsilon}^{\ell}\right)}\right)^{2+\lambda}\right] \\
& \quad+\frac{1}{\lambda+2} \frac{N_{r}}{4}\left(4|G|_{L^{2+\lambda}\left(\tilde{S}_{1+\varepsilon}\right)}\right)^{2+\lambda} .
\end{aligned}
$$

Let $K>0$ be defined by

$$
K=\max \left\{4\left|\sqrt[2+\lambda]{N_{z}} F_{r}\right|_{L^{2+\lambda}\left(\tilde{\gamma}_{1+\varepsilon}^{r}\right)}, 4\left|\sqrt[2+\lambda]{N_{z}} F_{\ell}\right|_{L^{2+\lambda}\left(\tilde{\gamma}_{1+\varepsilon}^{\ell}\right)}, 4|G|_{L^{2+\lambda}\left(\tilde{S}_{1+\varepsilon}\right)}\right\}
$$

and

Now (4.6) gives

$$
d=\max \left\{\frac{1}{2}, \frac{N_{r}}{4}\right\}
$$

$$
\frac{(1+\lambda) \kappa \varepsilon^{q}}{4\left(1+\frac{\lambda}{2}\right)^{2}} \int_{\tilde{P}_{1+\varepsilon}}\left|\theta_{+}^{\varepsilon}\right|^{2+\lambda} \leq \frac{3 d}{\lambda+2} K^{2+\lambda} .
$$

or

$$
\left|\theta_{+}^{\varepsilon}\right|_{L^{2+\lambda}\left(\tilde{P}_{1+\varepsilon}\right)} \leq\left(\frac{12 d\left(1+\frac{\lambda}{2}\right)^{2}}{(\lambda+2)(1+\lambda) \kappa \varepsilon^{q}}\right)^{\frac{1}{2+\lambda}} K .
$$


For $\lambda>3$ a simple calculation shows that

$$
\frac{\left(1+\frac{\lambda}{2}\right)^{2}}{(\lambda+2)(1+\lambda)}<\frac{1}{3}
$$

We end up with

$$
\left|\theta_{+}^{\varepsilon}\right|_{L^{2+\lambda}\left(\tilde{P}_{1+\varepsilon}\right)} \leq K\left(\frac{4 d}{\kappa \varepsilon^{q}}\right)^{\frac{1}{2+\lambda}}
$$

As $\lambda \rightarrow+\infty$ the left hand side converges to $\left|\theta_{+}^{\varepsilon}\right|_{L^{\infty}\left(\tilde{P}_{1+\varepsilon}\right)}$. The right-hand side converges to $K$. Thus

$$
\left|\theta_{+}^{\varepsilon}\right|_{L^{\infty}\left(\tilde{P}_{1+\varepsilon}\right)} \leq K
$$

For $q<0$, we deduce from $(4.13)$

$$
\begin{aligned}
& \frac{(1+\lambda)}{2\left(1+\frac{\lambda}{2}\right)^{2}}\left(\int_{\tilde{P}_{1+\varepsilon}}\left|\nabla\left[\left(\theta_{+}^{\varepsilon}\right)^{1+\frac{\lambda}{2}}\right]\right|^{2}+\int_{\tilde{S}_{1+\varepsilon}}\left|\left(\theta_{+}^{\varepsilon}\right)^{1+\frac{\lambda}{2}}\right|^{2}\right) \\
& \leq \frac{4^{1+\lambda}}{\lambda+2}\left[\left(\left|\sqrt[2+\lambda]{N_{z}} F_{r}\right|_{L^{2+\lambda}\left(\tilde{\gamma}_{1+\varepsilon}^{r}\right)}\right)^{2+\lambda}+\left(\left|\sqrt[2+\lambda]{N_{z}} F_{\ell}\right|_{L^{2+\lambda}\left(\tilde{\gamma}_{1+\varepsilon}^{\ell}\right)}\right)^{2+\lambda}\right] \\
& \quad+\frac{1}{\lambda+2} \frac{N_{r}}{4}\left(4|G|_{L^{2+\lambda}\left(\tilde{S}_{1+\varepsilon}\right)}\right)^{2+\lambda} .
\end{aligned}
$$

and, following the same steps, we get (4.17).

We now have the following a priori estimates:

Corollary 4.4. Let $q \in \mathbb{R}$ and let $\tilde{\theta}^{\varepsilon}$ be a weak solution to the problem (2.11)-(2.13). Suppose that (4.2) and (4.3) hold. Then, there exists $C>0$ such that

$$
\begin{aligned}
& \left|\tilde{\theta}^{\varepsilon}\right|_{L^{\infty}\left(\tilde{P}_{1+\varepsilon}\right)} \leq C, \\
& \left|\nabla \tilde{\theta}^{\varepsilon}\right|_{L^{2}\left(\tilde{P}_{1}\right)} \leq C, \\
& \left|\tilde{\theta}^{\varepsilon}\right|_{L^{2}\left(\tilde{S}_{1+\varepsilon}\right)} \leq C, \\
& \left|\tilde{\theta}^{\varepsilon}\right|_{L^{2}\left(\tilde{\gamma}_{1+\varepsilon}^{r}\right)} \leq C, \\
& \left|\tilde{\theta}^{\varepsilon}\right|_{L^{2}\left(\tilde{\gamma}_{1+\varepsilon}^{\ell}\right)} \leq C, \\
& \left|\nabla \tilde{\theta}^{\varepsilon}\right|_{L^{2}\left(\tilde{P}_{\varepsilon}\right)} \leq C \varepsilon^{-\frac{q}{2}} .
\end{aligned}
$$

Since we will take some limits in the weak formulation (6.16) we rewrite the estimates related to $\tilde{P}_{\varepsilon}$ from Corollary 4.4 in cylindrical coordinates.

Corollary 4.5. Let $q \in \mathbb{R}$ and let $\theta^{\varepsilon}$ be a weak solution to the problem (2.11)-(2.13). Then, there exists $C>0$ such that

$$
\left|\frac{\partial \theta^{\varepsilon}}{\partial r}\right|_{L^{2}\left(P_{\varepsilon}\right)},\left|\frac{\partial \theta^{\varepsilon}}{\partial \varphi}\right|_{L^{2}\left(P_{\varepsilon}\right)},\left|\frac{\partial \theta^{\varepsilon}}{\partial z}\right|_{L^{2}\left(P_{\varepsilon}\right)} \leq C \varepsilon^{-\frac{q}{2}} .
$$


Proof. The statements are consequence of change of variables and in particular since $r$ is bounded in $P_{\varepsilon}$, namely $1 \leq r \leq 1+\varepsilon \leq 2$.

Remark 4.6. The condition (4.3) is used here for the a priori estimate and later on for uniqueness of the solution to the effective problems. We could avoid any restriction on the Peclet number Pe by imposing the Dirichlet boundary condition on pipe's ends (at least on the left end). Roughly speaking, the Dirichlet condition corresponds to the case $\mathrm{N}_{z}^{f} \rightarrow \infty$.

\section{TWO-SCALE CONVERGENCE FOR THIN DOMAINS}

In this section we recall the definition and the basic properties of the two-scale convergence for thin domains. The method introduced in [9] (see also [10]) was designed for deriving effective physical laws in thin domains. We are dealing here with a junction of a thin domain with non-thin ones. The method has been applied previously on junction problems between two thin domains in [12]. We will define it for the thin domain which appears in our problem after change of variable by cylindrical coordinates $(r, \varphi, z)$, i.e. for domain $P_{\varepsilon}=\langle 1,1+\varepsilon\rangle \times\langle 0,2 \pi\rangle \times\langle 0, L\rangle$. For the definition of the two-scale convergence we will also need the rescaled version of this set

$$
P_{2-1}=\langle 1,2\rangle \times\langle 0,2 \pi\rangle \times\langle 0, L\rangle
$$

with variables $(\rho, \varphi, z)$. As usual, all functions given in cylindrical coordinates are assumed to be $2 \pi$-periodic with respect to $\varphi$.

Definition 5.1. We say that a sequence $\left\{v_{\varepsilon}\right\}_{\varepsilon>0}$, such that $v_{\varepsilon} \in L^{2}\left(P_{\varepsilon}\right)$, two- scale converges to a function $V \in L^{2}\left(P_{2-1}\right)$ (we use the notation 2 s convergence in the sequel) if

$$
\frac{1}{\varepsilon} \int_{P_{\varepsilon}} v_{\varepsilon}(r, \varphi, z) \phi\left(1+\frac{r-1}{\varepsilon}, \varphi, z\right) \mathrm{d} r \mathrm{~d} \varphi \mathrm{d} z \rightarrow \int_{P_{2-1}} V(\rho, \varphi, z) \phi(\rho, \varphi, z) \mathrm{d} \rho \mathrm{d} \varphi \mathrm{d} z
$$

for any $\phi \in L^{2}\left(P_{2-1}\right)$.

The definition is slightly modified compared to the one from [9], due to the curved geometry of our thin domain. We notice that the slow variable $r$ and the fast variable $\rho$ are related by formulas

$$
\rho=1+\frac{r-1}{\varepsilon} \Rightarrow r=1+\varepsilon(\rho-1) \Rightarrow \frac{r}{\varepsilon} \mathrm{d} r=\mathrm{d} \rho+O(\varepsilon) .
$$

For such convergence, we have the following compactness result (see [9], Thm. 1).

Theorem 5.2. (a) Let the sequence $\left\{v_{\varepsilon}\right\}_{\varepsilon>0}$ be such that $v_{\varepsilon} \in L^{2}\left(P_{\varepsilon}\right)$, and that

$$
\left|v_{\varepsilon}\right|_{L^{2}\left(P_{\varepsilon}\right)} \leq C \sqrt{\varepsilon}
$$

Then there exists function $V=V(\rho, \varphi, z) \in L^{2}\left(P_{2-1}\right)$ and a subsequence of $\left\{v_{\varepsilon}\right\}_{\varepsilon>0}$ (denoted, for simplicity, by the same symbol) such that

$$
v_{\varepsilon} \rightarrow V 2 s .
$$

(b) Let the sequence $\left\{v_{\varepsilon}\right\}_{\varepsilon>0}$ be such that $v_{\varepsilon} \in H^{1}\left(P_{\varepsilon}\right)$, and that

$$
\left|v_{\varepsilon}\right|_{H^{1}\left(P_{\varepsilon}\right)} \leq C \sqrt{\varepsilon}
$$

Then there exists a subsequence of $\left\{v_{\varepsilon}\right\}_{\varepsilon>0}$ (denoted, for simplicity, by the same symbol) and functions

$$
V=V(\varphi, z) \in \mathcal{H}=H^{1}(\langle 0,2 \pi\rangle \times\langle 0, L\rangle),
$$




$$
W \in \mathcal{Y}=\left\{W \in L^{2}\left(P_{2-1}\right) ; \frac{\partial W}{\partial \rho} \in L^{2}\left(P_{2-1}\right)\right\}
$$

such that

$$
\begin{aligned}
v_{\varepsilon} & \rightarrow V \quad 2 s, \\
\nabla v_{\varepsilon} & \rightarrow \nabla_{\varphi, z} V+\frac{\partial W}{\partial \rho} \mathbf{e}_{r} 2 s
\end{aligned}
$$

where

$$
\nabla_{\varphi, z} V=\frac{\partial V}{\partial z} \mathbf{e}_{z}+\frac{\partial V}{\partial \varphi} \mathbf{e}_{\varphi}
$$

is the tangential (intrinsic) gradient on the pipe's side.

(c) Let the sequence $\left\{v_{\varepsilon}\right\}_{\varepsilon>0}$ be such that $v_{\varepsilon} \in H^{1}\left(P_{\varepsilon}\right)$, and that

$$
\begin{aligned}
\left|v_{\varepsilon}\right|_{L^{2}\left(P_{\varepsilon}\right)} & \leq C \sqrt{\varepsilon}, \\
\varepsilon\left|\nabla v_{\varepsilon}\right|_{L^{2}\left(P_{\varepsilon}\right)} & \leq C \sqrt{\varepsilon} .
\end{aligned}
$$

Then there exist a subsequence of $\left\{v_{\varepsilon}\right\}_{\varepsilon>0}$ (denoted, for simpliity, by the same symbol) and function $W \in \mathcal{Y}$, such that

$$
\begin{aligned}
v_{\varepsilon} & \rightarrow W 2 s, \\
\varepsilon \nabla v_{\varepsilon} & \rightarrow \frac{\partial W}{\partial \rho} \mathbf{e}_{r} 2 s .
\end{aligned}
$$

We notice that our tangential gradient differs from the tangential part of the gradient in cylindrical coordinates

$$
\frac{\partial f}{\partial z} \mathbf{e}_{z}+\frac{1}{r} \frac{\partial f}{\partial \varphi} \mathbf{e}_{\varphi}
$$

It is due to the fact that on $\tilde{P}_{\varepsilon}$ we obviously have $r=1+O(\varepsilon)$. If we had a cylinder of radius $R$ there would be

$$
\frac{\partial W}{\partial z} \mathbf{e}_{z}+\frac{1}{R} \frac{\partial W}{\partial \varphi} \mathbf{e}_{\varphi}
$$

In the above text, instead of saying that $(\varphi, z) \in\langle 0,2 \pi\rangle \times\langle 0, L\rangle$ and imposing the periodicity with respect to $\varphi$, we could just say that it is defined on a side of a cylinder $S$. But that is a manifold and we would have to adapt the theory from [9] to curved geometry.

Lemma 5.3. Let $\left\{v_{\varepsilon}\right\}_{\varepsilon>0} \subset L^{2}\left(P_{\varepsilon}\right)$ two-scale converges to a function $V \in L^{2}\left(P_{2-1}\right)$. Then $r v_{\varepsilon} \rightarrow V$ two-scale, i.e.

$$
\frac{1}{\varepsilon} \int_{P_{\varepsilon}} v_{\varepsilon}(r, \varphi, z) \phi\left(1+\frac{r-1}{\varepsilon}, \varphi, z\right) r \mathrm{~d} r \mathrm{~d} \varphi \mathrm{d} z \rightarrow \int_{P_{2-1}} V(\rho, \varphi, z) \phi(\rho, \varphi, z) \mathrm{d} \rho \mathrm{d} \varphi \mathrm{d} z
$$

for any $\phi \in L^{2}\left(P_{2-1}\right)$.

Proof. Let $v_{\varepsilon} \rightarrow V$ two-scale. For a $\phi \in L^{2}\left(P_{2-1}\right)$ we consider

$$
\begin{aligned}
\frac{1}{\varepsilon} \int_{P_{\varepsilon}} & v_{\varepsilon}(r, \varphi, z) \phi\left(1+\frac{r-1}{\varepsilon}, \varphi, z\right) r \mathrm{~d} r \mathrm{~d} \varphi \mathrm{d} z \\
= & \frac{1}{\varepsilon} \int_{P_{\varepsilon}} v_{\varepsilon}(r, \varphi, z) \phi\left(1+\frac{r-1}{\varepsilon}, \varphi, z\right)\left(1+\frac{r-1}{\varepsilon}\right) \varepsilon \mathrm{d} r \mathrm{~d} \varphi \mathrm{d} z \\
& +\frac{1}{\varepsilon} \int_{P_{\varepsilon}} v_{\varepsilon}(r, \varphi, z) \phi\left(1+\frac{r-1}{\varepsilon}, \varphi, z\right)(1-\varepsilon) \mathrm{d} r \mathrm{~d} \varphi \mathrm{d} z .
\end{aligned}
$$

By a standard results for the two-scale convergence we have that $\left|\varepsilon v_{\varepsilon}\right|_{L^{2}\left(P_{\varepsilon}\right)} \rightarrow 0$, so the first term in the above equation tends to zero. For the second we apply the definition of two scale convergence and obtain the result of the lemma. 


\section{Convergence}

From the Corollaries 4.4 and 4.5 we deduce that there exists some $\tilde{\theta} \in H^{1}\left(\tilde{P}_{1}\right) \cap L^{\infty}\left(\tilde{P}_{1}\right)$, associated $\theta=$ $\tilde{\theta} \circ F \in L^{\infty}\left(P_{1}\right), \Theta \in L^{2}\left(P_{2-1}\right)$ and $T \in \mathcal{Y}$ such that, after possible extraction of a subsequence,

$$
\begin{aligned}
& \tilde{\theta}^{\varepsilon} \rightarrow \tilde{\theta} \quad \text { weak } * \text { in } L^{\infty}\left(\tilde{P}_{1}\right), \\
& \tilde{\theta}^{\varepsilon} \rightarrow \tilde{\theta} \quad \text { in } L^{2}\left(\tilde{P}_{1}\right), \\
& \nabla \tilde{\theta}^{\varepsilon} \rightarrow \nabla \tilde{\theta} \quad \text { weakly in } L^{2}\left(\tilde{P}_{1}\right), \\
& \left.\left.\tilde{\theta}^{\varepsilon}\right|_{\tilde{\gamma}_{1}^{\alpha}} \rightarrow \tilde{\theta}\right|_{\tilde{\gamma}_{1}^{\alpha}} \quad \text { in } L^{2}\left(\tilde{\gamma}_{1}^{\alpha}\right), \alpha=r, \ell, \\
& \theta^{\varepsilon} \rightarrow \Theta \quad 2 \mathrm{~s} \\
& \text { for } q \leq 1, \quad \varepsilon \nabla \theta^{\varepsilon} \rightarrow \frac{\partial \Theta}{\partial \rho} \mathbf{e}_{r} \quad 2 \mathrm{~s}, \quad \Theta \in \mathcal{H} \quad \& \quad T \in \mathcal{Y}, \\
& \text { for } q<1, \quad \varepsilon \nabla \theta^{\varepsilon} \rightarrow 0 \quad 2 \mathrm{~s} \quad \Rightarrow \Theta=\Theta(\varphi, z), \\
& \text { for } q \leq-1, \quad \nabla \theta^{\varepsilon} \rightarrow \nabla \nabla_{\varphi z} \Theta+\frac{\partial T}{\partial \rho} \mathbf{e}_{r} \quad 2 \mathrm{~s}, \quad \Theta \in \mathcal{Y}, \\
& \text { for } q<-1, \quad \nabla \theta^{\varepsilon} \rightarrow 0 \quad 2 \mathrm{~s} \quad \Rightarrow \Theta=\text { const. }
\end{aligned}
$$

Furthermore, as the following theorem gives, for $q \leq 1$ on the contact of the fluid and the pipe's wall we obtain the continuity of the temperature.

Theorem 6.1. Let $q \leq 1, \tilde{\theta} \in H^{1}\left(\tilde{P}_{1}\right)$ be the weak limit from (6.1)-(6.3) and let $\Theta$ be the two-scale limit from (6.5). Then

$$
\Theta(1, \varphi, z)=\theta(1, \varphi, z) \text { in } L^{2}(\langle 0,2 \pi\rangle \times\langle 0, L\rangle) .
$$

Here $\theta(1, \varphi, z)$ and $\Theta(1, \varphi, z)$ denote the traces.

Proof. Let $\phi_{0}$ be a smooth function defined in $P_{1}$ and let $\phi_{1}$ be a smooth function defined in $P_{2-1}$ such that $\phi_{1}(2, \varphi, z)=0$. Furthermore let

$$
\phi_{0}(1, \varphi, z)=\phi_{1}(1, \varphi, z)
$$

We define

$$
\Phi(r, \varphi, z)= \begin{cases}\phi_{0}(r, \varphi, z), & (r, \varphi, z) \in P_{1}, \\ \phi_{1}\left(1+\frac{r-1}{\varepsilon}, \varphi, z\right), & (r, \varphi, z) \in P_{\varepsilon} .\end{cases}
$$

This function is admissible test function so we get

$$
0=\int_{P_{1+\varepsilon}} \partial_{r}\left(\theta^{\varepsilon} \Phi r\right) \mathrm{d} r \mathrm{~d} \varphi \mathrm{d} z .
$$

Calculating the partial derivative we obtain

$$
\begin{aligned}
0= & \int_{P_{1}}\left(\partial_{r} \theta^{\varepsilon} \phi_{0} r+\theta^{\varepsilon} \partial_{r} \phi_{0} r+\theta^{\varepsilon} \phi_{0}\right) \mathrm{d} r \mathrm{~d} \varphi \mathrm{d} z \\
& +\int_{P_{\varepsilon}}\left(\partial_{r} \theta^{\varepsilon}(r, \varphi, z) \phi_{1}\left(1+\frac{r-1}{\varepsilon}, \varphi, z\right) r\right. \\
& +\theta^{\varepsilon}(r, \varphi, z) \frac{1}{\varepsilon} \partial_{\rho} \phi_{1}\left(1+\frac{r-1}{\varepsilon}, \varphi, z\right) r \\
& \left.+\theta^{\varepsilon}(r, \varphi, z) \phi_{1}\left(1+\frac{r-1}{\varepsilon}, \varphi, z\right)\right) \mathrm{d} r \mathrm{~d} \varphi \mathrm{d} z
\end{aligned}
$$


Now we use convergences for $q \leq 1$ and take the limit in each term to obtain

$$
0=\int_{P_{1}}\left(\partial_{r} \theta \phi_{0} r+\theta \partial_{r} \phi_{0} r+\theta \phi_{0}\right) \mathrm{d} r \mathrm{~d} \varphi \mathrm{d} z+\int_{P_{2-1}}\left(\partial_{\rho} \Theta \phi_{1}+\Theta \partial_{\rho} \phi_{1}\right) \mathrm{d} \rho \mathrm{d} \varphi \mathrm{d} z .
$$

This implies

$$
0=\int_{P_{1}} \partial_{r}\left(\theta \phi_{0} r\right) \mathrm{d} r \mathrm{~d} \varphi \mathrm{d} z+\int_{P_{2-1}} \partial_{\rho}\left(\Theta \phi_{1}\right) \mathrm{d} \rho \mathrm{d} \varphi \mathrm{d} z
$$

and therefore, using the properties of test functions $\phi_{0}$ and $\phi_{1}$ we obtain

$$
0=\int_{S}\left(\theta(1, \varphi, z) \phi_{0}(1, \varphi, z)-\Theta(1, \varphi, z) \phi_{1}(1, \varphi, z)\right) \mathrm{d} \varphi \mathrm{d} z
$$

Arbitrariness of test functions implies the statement of the theorem.

\subsection{Case $q<-1$}

In this case the diffusion through pipe's wall is very strong. In fact, so strong that the temperature is constant in the whole wall. However this constant is not prescribed, but depends on the solution. Furthermore, we get an interesting non-local boundary condition on the wall of the pipe. Basically it says that the total temperature flux through the wall of the pipe is proportional to the difference of the average exterior temperature and the temperature on the wall (which is constant). More precisely, we get the following result.

Theorem 6.2. Let $q<-1$ and let $\theta^{\varepsilon} \in H^{1}\left(P_{1+\varepsilon}\right)$ be the solution to the problem (2.11)-(2.13). Suppose that the conditions (4.2) and (4.3) hold. Then

$$
\begin{aligned}
& \tilde{\theta}^{\varepsilon} \rightarrow \tilde{\theta} \quad \text { weak } * \text { in } L^{\infty}\left(\tilde{P}_{1}\right), \\
& \tilde{\theta}^{\varepsilon} \rightarrow \tilde{\theta} \text { weakly in } H^{1}\left(\tilde{P}_{1}\right) \text {, }
\end{aligned}
$$

where $\tilde{\theta} \in H^{1}\left(\tilde{P}_{1}\right) \cap L^{\infty}\left(\tilde{P}_{1}\right)$ is the unique solution to the problem

$$
\begin{array}{lr}
-\Delta \tilde{\theta}+\operatorname{Pe} \tilde{v} \frac{\partial \tilde{\theta}}{\partial z}=0 & \text { in } \tilde{P}_{1}, \\
-\frac{\partial \tilde{\theta}}{\partial z}+\operatorname{Pe} \tilde{v} \tilde{\theta}=N_{z}^{f}\left(\tilde{F}_{\ell}-\tilde{\theta}\right) & \text { on } \tilde{\gamma}_{1}^{\ell}, \\
\frac{\partial \tilde{\theta}}{\partial z}-\operatorname{Pe} \tilde{v} \tilde{\theta}=N_{z}^{f}\left(\tilde{F}_{r}-\tilde{\theta}\right) & \text { on } \tilde{\gamma}_{1}^{r}, \\
\tilde{\theta}=\text { const. } & \text { on } \tilde{S}_{1}, \\
\frac{\partial \bar{\theta}}{\partial r}(1)=N_{r}(\bar{G}-\bar{\theta}(1)), &
\end{array}
$$

where

$$
\begin{aligned}
\bar{G} & =\frac{1}{2 \pi L} \int_{S} G(\varphi, z) \mathrm{d} \varphi \mathrm{d} z, \\
\bar{\theta}(r) & =\frac{1}{2 \pi L} \int_{S} \theta(r, \varphi, z) \mathrm{d} \varphi \mathrm{d} z .
\end{aligned}
$$


Proof. First of all, we know from the convergence (6.9) and Theorem 6.1 that the weak limit $\tilde{\theta}$ is constant at the boundary $\tilde{S}_{1}$. Thus it belongs to

$$
\mathcal{V}_{\langle-\infty,-1\rangle}=\left\{\tilde{\phi} \in H^{1}\left(\tilde{P}_{1}\right) ;\left.\tilde{\phi}\right|_{r=1}=\text { const }\right\} .
$$

Let us denote $\tilde{\theta}_{0}=\left.\tilde{\theta}\right|_{r=1}$. Then we take $\tilde{\psi} \in \mathcal{V}_{\langle-\infty,-1\rangle}$, similarly denote $\tilde{\psi}_{0}=\left.\tilde{\psi}\right|_{r=1} \in \mathbb{R}$ and define

$$
\tilde{\Psi}= \begin{cases}\tilde{\psi} & \text { in } \tilde{P}_{1} \\ \tilde{\psi}_{0} & \text { in } \tilde{P}_{\varepsilon} .\end{cases}
$$

For $\Psi$ as a test function, the weak formulation (2.14) reads

$$
\begin{aligned}
\int_{\tilde{P}_{1}} & \nabla \tilde{\theta}^{\varepsilon} \cdot \nabla \tilde{\Psi} \mathrm{d} V-\operatorname{Pe} \int_{\tilde{P}_{1}} \tilde{v} \tilde{\theta}^{\varepsilon} \frac{\partial \tilde{\Psi}}{\partial z} \mathrm{~d} V \\
& +\int_{\tilde{\gamma}_{1+\varepsilon}^{e}} N_{z} \tilde{\theta}^{\varepsilon} \tilde{\Psi} \mathrm{d} a+\int_{\tilde{\gamma}_{1+\varepsilon}^{r}} N_{z} \tilde{\theta}^{\varepsilon} \tilde{\Psi} \mathrm{d} a+N_{r} \int_{\tilde{S}_{1+\varepsilon}} \tilde{\theta}^{\varepsilon} \tilde{\psi}_{0} \mathrm{~d} a \\
= & \int_{\tilde{\gamma}_{1+\varepsilon}^{\ell}} N_{z} \tilde{F}_{\ell} \tilde{\Psi} \mathrm{d} a+\int_{\tilde{\gamma}_{1+\varepsilon}^{r}} N_{z} \tilde{F}_{r} \tilde{\Psi} \mathrm{d} a+N_{r} \int_{\tilde{S}_{1+\varepsilon}} \tilde{G} \tilde{\psi}_{0} \mathrm{~d} a .
\end{aligned}
$$

Now we take the limit in this equation and use the convergences for $\theta^{\varepsilon}$ and $\tilde{\theta}^{\varepsilon}$. For the first two terms on the left hand side we use (6.3) and (6.1). For the third and the fourth term in the left hand side we use estimates (4.21) and (4.22) to obtain for $\alpha \in\{l, r\}$

$$
\left.\left|\int_{\tilde{\gamma}_{1+\varepsilon}^{\alpha} \backslash \tilde{\gamma}_{1}^{\alpha}} N_{z} \tilde{\theta}^{\varepsilon} \tilde{\Psi} \mathrm{d} a\right| \leq N_{z, \max }\left|\tilde{\theta}^{\varepsilon}\right|_{L^{2}\left(\tilde{\gamma}_{1+\varepsilon}^{\alpha}\right)}\right)\left.\tilde{\psi}_{0}\right|_{L^{2}\left(\tilde{\gamma}_{1+\varepsilon}^{\alpha} \backslash \tilde{\gamma}_{1}^{\alpha}\right)} \leq C \varepsilon .
$$

Thus application of (6.4) implies

$$
\int_{\tilde{\gamma}_{1+\varepsilon}^{\alpha}} N_{z} \tilde{\theta}^{\varepsilon} \tilde{\Psi} \mathrm{d} a \rightarrow N_{z}^{f} \int_{\tilde{\gamma}_{1}^{\alpha}} \tilde{\theta} \tilde{\Psi} \mathrm{d} a, \quad \alpha \in\{l, r\} .
$$

Similarly we obtain

$$
\int_{\tilde{\gamma}_{1+\varepsilon}^{\alpha}} \tilde{F}_{\alpha} \tilde{\Psi} \mathrm{d} a \rightarrow \int_{\tilde{\gamma}_{1}^{\alpha}} \tilde{F}_{\alpha} \tilde{\Psi} \mathrm{d} a, \quad \alpha \in\{l, r\} .
$$

For the last term on the left hand side we argue as follows

$$
\begin{aligned}
\int_{\tilde{S}_{1+\varepsilon}} \tilde{\theta}^{\varepsilon} \tilde{\psi}_{0} \mathrm{~d} a & =\tilde{\psi}_{0}(1+\varepsilon) \int_{S} \theta^{\varepsilon}(1+\varepsilon, \varphi, z) \mathrm{d} \varphi \mathrm{d} z \\
& =\tilde{\psi}_{0}(1+\varepsilon) \int_{S}\left(\theta^{\varepsilon}(1, \varphi, z)+\int_{1}^{1+\varepsilon} \frac{\partial \theta^{\varepsilon}}{\partial r}(r, \varphi, z) \mathrm{d} r\right) \mathrm{d} \varphi \mathrm{d} z \\
& =\tilde{\psi}_{0}(1+\varepsilon)\left(\int_{\tilde{S}_{1}} \tilde{\theta}^{\varepsilon} \mathrm{d} a+\left|\frac{\partial \theta^{\varepsilon}}{\partial r}\right|_{L^{1}\left(P_{\varepsilon}\right)}\right) .
\end{aligned}
$$

By (4.24) we have that $\left|\frac{\partial \theta^{\varepsilon}}{\partial r}\right|_{L^{1}\left(P_{\varepsilon}\right)} \leq C\left|\frac{\partial \theta^{\varepsilon}}{\partial r}\right|_{L^{2}\left(P_{\varepsilon}\right)} \sqrt{\varepsilon} \leq C \varepsilon$ for $q<-1$, so using (6.2) and (6.3) we obtain

$$
\int_{\tilde{S}_{1+\varepsilon}} \tilde{\theta}^{\varepsilon} \tilde{\psi}_{0} \mathrm{~d} a \rightarrow \tilde{\psi}_{0} \int_{\tilde{S}_{1}} \tilde{\theta} \mathrm{d} a=2 \pi L \tilde{\psi}_{0} \tilde{\theta}_{0}
$$


Similar argument gives

$$
\int_{\tilde{S}_{1+\varepsilon}} \tilde{G} \tilde{\psi}_{0} \mathrm{~d} a=\tilde{\psi}_{0}(1+\varepsilon) \int_{S} G(\varphi, z) \mathrm{d} \varphi \mathrm{d} z \rightarrow \tilde{\psi}_{0} \int_{S} G(\varphi, z) \mathrm{d} \varphi \mathrm{d} z=2 \pi \tilde{\psi}_{0} \bar{G} .
$$

Collecting all these convergences in (6.16) we obtain

$$
\begin{aligned}
\int_{\tilde{P}_{1}} & \nabla \tilde{\theta} \cdot \nabla \tilde{\psi} \mathrm{d} V-\operatorname{Pe} \int_{\tilde{P}_{1}} \tilde{v} \tilde{\theta} \frac{\partial \tilde{\psi}}{\partial z} \mathrm{~d} V \\
& +N_{z}^{f} \int_{\tilde{\gamma}_{1}^{\ell}} \tilde{\theta} \tilde{\psi} \mathrm{d} a+N_{z}^{f} \int_{\tilde{\gamma}_{1}^{r}} \tilde{\theta} \tilde{\psi} \mathrm{d} a+N_{r} 2 \pi L \tilde{\theta}_{0} \tilde{\psi}_{0} \\
= & N_{z}^{f} \int_{\tilde{\gamma}_{1}^{\ell}} \tilde{F}_{\ell} \tilde{\psi} \mathrm{d} a+N_{z}^{f} \int_{\tilde{\gamma}_{1}^{r}} \tilde{F}_{r} \psi \mathrm{d} a+N_{r} 2 \pi \bar{G}_{0} .
\end{aligned}
$$

The existence of solution of the two-scale problem has been proved by the limiting procedure (although it could be proved by the Lax \& Milgram theorem on an appropriate two scale space). The uniqueness, however, has to be proved. Since the problem is linear it is standard procedure to assume the existence of two solutions $\tilde{\theta}_{1}$ and $\tilde{\theta}_{2}$ and to look at their difference $U=\tilde{\theta}_{1}-\tilde{\theta}_{2}$. Then $U_{0}=\left.\tilde{\theta}_{1}\right|_{r=1}-\left.\tilde{\theta}_{2}\right|_{r=1}=$ const. Now we use that same $U$ for the test function in (6.17). It leads to

$$
\int_{\tilde{P}_{1}}|\nabla U|^{2} \mathrm{~d} V-\operatorname{Pe} \int_{\tilde{P}_{1}} \tilde{v} U \frac{\partial U}{\partial z} \mathrm{~d} V+N_{z}^{f} \int_{\tilde{\gamma}_{1}^{\ell}} U^{2} \mathrm{~d} a+N_{z}^{f} \int_{\tilde{\gamma}_{1}^{r}} U^{2} \mathrm{~d} a+N_{r} 2 \pi L U_{0}^{2}=0 .
$$

A simple computation gives

$$
-\operatorname{Pe} \int_{\tilde{P}_{1}} \tilde{v} U \frac{\partial U}{\partial z} \mathrm{~d} V=-\frac{\operatorname{Pe}}{2} \int_{\tilde{P}_{1}} \tilde{v} \frac{\partial U^{2}}{\partial z} \mathrm{~d} V=\frac{\operatorname{Pe}}{2}\left(\int_{\tilde{\gamma}_{1}^{e}} U^{2} \mathrm{~d} a-\int_{\tilde{\gamma}_{1}^{r}} U^{2} \mathrm{~d} a\right) .
$$

So that (using (4.3))

$$
-\operatorname{Pe} \int_{\tilde{P}_{1}} \tilde{v} U \frac{\partial U}{\partial z} \mathrm{~d} V+N_{z}^{f} \int_{\tilde{\gamma}_{1}^{r}} U^{2} \mathrm{~d} a \geq\left(N_{z}^{f}-\frac{\mathrm{Pe}}{\pi}\right) \int_{\tilde{\gamma}_{1}^{r}} U^{2} \mathrm{~d} a \geq 0 .
$$

Now we have

$$
\int_{\tilde{P}_{1}}|\nabla U|^{2} \mathrm{~d} V+N_{z}^{f} \int_{\tilde{\gamma}_{1}^{\ell}} U^{2} \mathrm{~d} a=0
$$

implying that $U=0$. Therefore the problem: find $\tilde{\theta} \in \mathcal{V}_{\langle-\infty,-1\rangle}$ such that (6.17) holds for any $\tilde{\psi} \in \mathcal{V}_{\langle-\infty,-1\rangle}$ has a unique solution, meaning that the whole family

$$
\tilde{\theta}^{\varepsilon} \rightarrow \tilde{\theta} \quad \text { weakly in } \quad H^{1}\left(\tilde{P}_{1}\right)
$$

and not just a subsequence.

The decoupling gives (6.11)-(6.13).

\subsection{Case $q=-1$}

In this case the processes in the fluid and in the pipe's wall are of the same order and can both be seen in the effective model. We obtain the coupled model consisting of the convection-diffusion equation in the fluid and the Laplace-Beltrami equation on the $2 \mathrm{D}$ surface representing the pipe's wall, coupled via the continuity of the temperature. 
Theorem 6.3. Let $q=-1$ and let $\tilde{\theta}^{\varepsilon} \in H^{1}\left(\tilde{P}_{1+\varepsilon}\right)$ be the weak solution to the problem (2.11)-(2.13). Suppose that (4.2) and (4.3) hold. Then

$$
\begin{aligned}
& \tilde{\theta}^{\varepsilon} \rightarrow \tilde{\theta} \quad \text { weak } * \text { in } \quad L^{\infty}\left(\tilde{P}_{1}\right), \\
& \tilde{\theta}^{\varepsilon} \rightarrow \tilde{\theta} \text { weakly in } H^{1}\left(\tilde{P}_{1}\right), \\
& \theta^{\varepsilon} \rightarrow \Theta \quad 2 \mathrm{~s}
\end{aligned}
$$

where $(\tilde{\theta}, \Theta) \in\left[H^{1}\left(\tilde{P}_{1}\right) \cap L^{\infty}\left(\tilde{P}_{1}\right)\right] \times \mathcal{H}$ is the unique solution to the two-scale problem (6.21). Furthermore

$$
\theta(1, \varphi, z)=\Theta(\varphi, z)
$$

in the sense of traces, and the problem (6.21) is equivalent to the decoupled system

$$
\begin{array}{ll}
-\Delta \tilde{\theta}+\operatorname{Pe} \tilde{v} \frac{\partial \tilde{\theta}}{\partial z}=0 & \text { in } \tilde{P}_{1}, \\
-\frac{\partial \tilde{\theta}}{\partial z}+\operatorname{Pe} \tilde{v} \tilde{\theta}=N_{z}^{f}\left(\tilde{F}_{\ell}-\tilde{\theta}\right) & \text { on } \tilde{\gamma}_{1}^{\ell}, \\
\frac{\partial \tilde{\theta}}{\partial z}-\operatorname{Pe} \tilde{v} \tilde{\theta}=N_{z}^{f}\left(\tilde{F}_{r}-\tilde{\theta}\right) & \text { on } \tilde{\gamma}_{1}^{r}, \\
\frac{\partial \tilde{\theta}}{\partial r}-\kappa \Delta_{S} \tilde{\theta}+N_{r} \tilde{\theta}=N_{r} \tilde{G} & \text { in } \tilde{S}_{1}, \\
\left.\frac{\partial \tilde{\theta}}{\partial z}\right|_{r=1}=0 & \text { for } z=0, L .
\end{array}
$$

Here

$$
\left(\Delta_{S} \tilde{\theta}\right) \circ F=\frac{\partial^{2} \theta}{\partial \varphi^{2}}+\frac{\partial^{2} \theta}{\partial z^{2}}
$$

and $\tilde{S}_{1}$ is the side (curved part of the boundary) of the cylinder $\tilde{P}_{1}$.

Proof. We proceed as in the previous section with the difference that now we take

$$
\tilde{\psi} \in \mathcal{V}_{-1}=\left\{\tilde{\phi} \in H^{1}\left(\tilde{P}_{1}\right) ; \phi(1, \cdot)=\tilde{\phi} \circ F(1, \cdot) \in H^{1}(\langle 0,2 \pi\rangle \times\langle 0, L\rangle)\right\} .
$$

We then put, as before

$$
\tilde{\Psi}= \begin{cases}\tilde{\psi} & \text { in } \tilde{P}_{1} \\ \left.\tilde{\psi}\right|_{r=1} & \text { in } \tilde{P}_{\varepsilon}\end{cases}
$$

Let us denote $\psi=\tilde{\psi} \circ F$. The only difference comparing with (6.16) are in the integrals over $\tilde{P}_{\varepsilon}$ and $\tilde{S}_{1+\varepsilon}$. To treat the integral over $\tilde{P}_{\varepsilon}$ we use the two-scale convergence (6.8) giving

$$
\begin{aligned}
\frac{1}{\varepsilon} \int_{P_{\varepsilon}} & \left(r \frac{\partial \theta^{\varepsilon}}{\partial r} \frac{\partial \tilde{\Psi}}{\partial r}+\frac{1}{r} \frac{\partial \theta^{\varepsilon}}{\partial \varphi} \frac{\partial \tilde{\Psi}}{\partial \varphi}+r \frac{\partial \theta^{\varepsilon}}{\partial z} \frac{\partial \tilde{\Psi}}{\partial z}\right) \mathrm{d} r \mathrm{~d} \varphi \mathrm{d} z \\
\quad & =\frac{1}{\varepsilon} \int_{P_{\varepsilon}}\left(\frac{1}{r} \frac{\partial \theta^{\varepsilon}}{\partial \varphi}(r, \varphi, z) \frac{\partial \psi}{\partial \varphi}(1, \varphi, z)+r \frac{\partial \theta^{\varepsilon}}{\partial z}(r, \varphi, z) \frac{\partial \psi}{\partial z}(1, \varphi, z)\right) \mathrm{d} r \mathrm{~d} \varphi \mathrm{d} z \\
& \rightarrow \int_{P_{2-1}}\left(\frac{\partial \Theta}{\partial \varphi}(\varphi, z) \frac{\partial \psi}{\partial \varphi}(1, \varphi, z)+\frac{\partial \Theta}{\partial z}(\varphi, z) \frac{\partial \psi}{\partial z}(1, \varphi, z)\right) \mathrm{d} z \mathrm{~d} \varphi \\
= & \int_{\tilde{S}_{1}} \nabla_{\tau} \tilde{\theta} \cdot \nabla_{\tau} \tilde{\psi} \mathrm{d} a
\end{aligned}
$$


where $\nabla_{\tau}$ denotes the tangential gradient. It can be expressed by intrinsic gradient through $\nabla_{\tau} \tilde{\theta} \circ F=\nabla_{\varphi, z} \theta$. That produces one additional term in the limit problem.

For the treatment of the integrals over $\tilde{S}_{1+\varepsilon}$ we do as follows:

$$
\begin{aligned}
\int_{\tilde{S}_{1+\varepsilon}} \tilde{\theta}^{\varepsilon} \tilde{\Psi} \mathrm{d} a & =(1+\varepsilon) \int_{S} \theta^{\varepsilon}(1+\varepsilon, \varphi, z) \psi(1, \varphi, z) \mathrm{d} \varphi \mathrm{d} z \\
& =(1+\varepsilon) \int_{S} \psi(1, \varphi, z)\left(\theta^{\varepsilon}(1, \varphi, z)+\int_{1}^{1+\varepsilon} \frac{\partial \theta^{\varepsilon}}{\partial r}(r, \varphi, z) \mathrm{d} r\right) \mathrm{d} \varphi \mathrm{d} z \\
& =(1+\varepsilon)\left(\int_{S} \theta^{\varepsilon}(1, \varphi, z) \psi(1, \varphi, z) \mathrm{d} \varphi \mathrm{d} z+\left|\frac{\partial \theta^{\varepsilon}}{\partial r}\right|_{L^{2}\left(P_{\varepsilon}\right)}|\psi|_{L^{2}\left(P_{\varepsilon}\right)}\right) .
\end{aligned}
$$

Since $\left|\frac{\partial \theta^{\varepsilon}}{\partial r}\right|_{L^{2}\left(P_{\varepsilon}\right)} \leq C \varepsilon^{1 / 2}$ by (4.24) and $|\psi|_{L^{2}\left(P_{\varepsilon}\right)} \leq C \varepsilon^{1 / 2}$ using the convergences (6.1) and (6.3) together with the trace theorem we obtain

$$
\int_{\tilde{S}_{1+\varepsilon}} \tilde{\theta}^{\varepsilon} \tilde{\Psi} \mathrm{d} a \rightarrow \int_{S} \theta(1, \varphi, z) \psi(1, \varphi, z) \mathrm{d} \varphi \mathrm{d} z=\int_{\tilde{S}_{1}} \theta \psi \mathrm{d} a .
$$

The two-scale problem now reads: find $\tilde{\theta} \in \mathcal{V}_{-1}$ such that

$$
\begin{aligned}
\int_{\tilde{P}_{1}} & \nabla \tilde{\theta} \cdot \nabla \tilde{\psi} \mathrm{d} V-\operatorname{Pe} \int_{\tilde{P}_{1}} \tilde{v} \tilde{\theta} \frac{\partial \tilde{\psi}}{\partial z} \mathrm{~d} V+\kappa \int_{\tilde{S}_{1}} \nabla_{\tau} \tilde{\theta} \cdot \nabla_{\tau} \tilde{\psi} \mathrm{d} a \\
& +N_{z}^{f} \int_{\tilde{\gamma}_{1}^{e}} \tilde{\theta} \tilde{\psi} \mathrm{d} a+N_{z}^{f} \int_{\tilde{\gamma}_{1}^{r}} \tilde{\theta} \tilde{\psi} \mathrm{d} a+N_{r} \int_{\tilde{S}_{1}} \tilde{\theta} \tilde{\psi} \mathrm{d} a \\
= & N_{z}^{f} \int_{\tilde{\gamma}_{1}^{\ell}} \tilde{F}_{\ell} \tilde{\psi} \mathrm{d} a+N_{z}^{f} \int_{\tilde{\gamma}_{1}^{r}} \tilde{F}_{r} \tilde{\psi} \mathrm{d} a+N_{r} \int_{\tilde{S}_{1}} \tilde{G} \tilde{\psi} \mathrm{d} a
\end{aligned}
$$

holds for any $\psi \in \mathcal{V}_{-1}$.

Like in the previous case $q<-1$, the existence follows from the limiting procedure, and the proof of uniqueness is analogous. Indeed, assuming that we have two solutions $\tilde{\theta}_{1}$ and $\tilde{\theta}_{2}$ and taking the difference $U=\tilde{\theta}_{1}-\tilde{\theta}_{2}$, we find that $U$ satisfies

$$
\begin{aligned}
& \int_{\tilde{P}_{1}}|\nabla U|^{2} \mathrm{~d} V-\operatorname{Pe} \int_{\tilde{P}_{1}} \tilde{v} U \frac{\partial U}{\partial z} \mathrm{~d} V+\kappa \int_{\tilde{S}_{1}}\left|\nabla_{\tau} U\right|^{2} \mathrm{~d} a \\
& \quad+N_{z}^{f} \int_{\tilde{\gamma}_{1}^{e}} U^{2} \mathrm{~d} a+N_{z}^{f} \int_{\tilde{\gamma}_{1}^{r}} U^{2} \mathrm{~d} a+N_{r} \int_{\tilde{S}_{1}} U^{2} \mathrm{~d} a=0 .
\end{aligned}
$$

Repeating the same procedure as in case $q<-1$, and using the condition (4.3) implies $U=0$.

Uniqueness of the solution, implies that the whole family

$$
\tilde{\theta}^{\varepsilon} \rightarrow \tilde{\theta} \quad \text { weakly in } H^{1}\left(\tilde{P}_{1}\right)
$$

and not just a subsequence.

To decouple the two-scale problem we first easily conclude that

$$
-\Delta \tilde{\theta}+\operatorname{Pe} \tilde{v} \frac{\partial \tilde{\theta}}{\partial z}=0 \quad \text { in } \quad \tilde{P}_{1}
$$

in the sense of distributions. On the other hand, the boundary condition on the pipes wall $r=1$ is of the second order and contains the Laplace-Beltrami operator

$$
\frac{\partial \tilde{\theta}}{\partial r}-\kappa \Delta_{S} \tilde{\theta}+N_{r} \tilde{\theta}=N_{r} \tilde{G} \quad \text { on } \quad \tilde{S}_{1},
$$


with

$$
\left(\Delta_{S} \tilde{\theta}\right) \circ F=\frac{\partial^{2} \theta}{\partial \varphi^{2}}+\frac{\partial^{2} \theta}{\partial z^{2}}
$$

\subsection{Case $-1<q<1$}

This is the case when we obtain the model that is usually found in the engineering literature. The wall of the pipe is not present in the effective model and we have the Robin boundary condition between the fluid in the pipe and the surrounding medium, as if the wall was not there.

Theorem 6.4. Let $-1<q<1$ and let $\tilde{\theta}^{\varepsilon} \in H^{1}\left(\tilde{P}_{1+\varepsilon}\right)$ be a weak solution to the problem (2.11)-(2.13) and let the data satisfy (4.2). Furthermore, let $\mathrm{Pe}$ and $N_{z}^{f}$ be such that (4.3) holds. Then

$$
\begin{array}{lll}
\tilde{\theta}^{\varepsilon} \rightarrow \tilde{\theta} & \text { weak * in } & L^{\infty}\left(\tilde{P}_{1}\right), \\
\tilde{\theta}^{\varepsilon} \rightarrow \tilde{\theta} & \text { weakly in } & H^{1}\left(\tilde{P}_{1}\right),
\end{array}
$$

where $\tilde{\theta} \in H^{1}\left(\tilde{P}_{1}\right) \cap L^{\infty}\left(\tilde{P}_{1}\right)$ is the unique solution to the problem

$$
\begin{array}{lr}
-\Delta \tilde{\theta}+\operatorname{Pe} \tilde{v} \frac{\partial \tilde{\theta}}{\partial z}=0 & \text { in } \tilde{P}_{1}, \\
-\frac{\partial \tilde{\theta}}{\partial z}+\operatorname{Pe} \tilde{v} \tilde{\theta}=N_{z}^{f}\left(\tilde{F}_{\ell}-\tilde{\theta}\right) & \text { on } \tilde{\gamma}_{1}^{\ell}, \\
\frac{\partial \tilde{\theta}}{\partial z}-\operatorname{Pe} \tilde{v} \tilde{\theta}=N_{z}^{f}\left(\tilde{F}_{r}-\tilde{\theta}\right) & \text { on } \tilde{\gamma}_{1}^{r}, \\
\frac{\partial \tilde{\theta}}{\partial r}=N_{r}(\tilde{G}-\tilde{\theta}) & \text { on } \tilde{S}_{1} .
\end{array}
$$

Proof. The test function is constructed like in the proofs of Theorems 6.2 and 6.3 . The passage to the limit is also the same as in the proofs of Theorems 6.2 and 6.3 , with the only difference in the integral over $\tilde{P}_{\varepsilon}$. In this case for a test function $\tilde{\Psi} \in C^{1}\left(\tilde{P}_{1+\varepsilon}\right)$ and using estimate (4.23) we have

$$
\varepsilon^{q} \kappa \int_{\tilde{P}_{\varepsilon}} \nabla \tilde{\theta}^{\varepsilon} \cdot \nabla \tilde{\Psi} \mathrm{d} V \leq C \varepsilon^{q}\left|\nabla \tilde{\theta}^{\varepsilon}\right|_{L^{2}\left(\tilde{P}_{\varepsilon}\right)}|\nabla \tilde{\Psi}|_{L^{\infty}\left(\tilde{P}_{\varepsilon}\right)}\left|\tilde{P}_{\varepsilon}\right|^{\frac{1}{2}} \leq C \varepsilon^{\frac{1+q}{2}} \rightarrow 0
$$

Thus the limit problem now reads: find $\tilde{\theta} \in \mathcal{V}_{\langle-1,1\rangle}:=H^{1}\left(\tilde{P}_{1}\right)$ such that

$$
\begin{aligned}
\int_{\tilde{P}_{1}} & \nabla \tilde{\theta} \cdot \nabla \tilde{\psi} \mathrm{d} V-\operatorname{Pe} \int_{\tilde{P}_{1}} \tilde{v} \tilde{\theta} \frac{\partial \tilde{\psi}}{\partial z} \mathrm{~d} V \\
& +N_{z}^{f} \int_{\tilde{\gamma}_{1}^{\ell}} \tilde{\theta} \tilde{\psi} \mathrm{d} a+N_{z}^{f} \int_{\tilde{\gamma}_{1}^{r}} \tilde{\theta} \tilde{\psi} \mathrm{d} a+N_{r} \int_{\tilde{S}_{1}} \tilde{\theta} \tilde{\psi} \mathrm{d} a \\
= & N_{z}^{f} \int_{\tilde{\gamma}_{1}^{e}} \tilde{F}_{\ell} \tilde{\psi} \mathrm{d} a+N_{z}^{f} \int_{\tilde{\gamma}_{1}^{r}} \tilde{F}_{r} \psi \mathrm{d} a+N_{r} \int_{\tilde{S}_{1}} \tilde{G} \tilde{\psi} \mathrm{d} a
\end{aligned}
$$

for all $\tilde{\psi} \in C^{1}\left(\tilde{P}_{1}\right)$ (which is the restriction of $\tilde{\Psi}$ on $\left.\tilde{P}_{1}\right)$. Since $C^{1}\left(\tilde{P}_{1}\right)$ is dense in $\mathcal{V}_{\langle-1,1\rangle}$ we obtain the weak formulation of (6.23) and (6.24). Uniqueness can be proved as in the previous cases and the conclusions follow easily. 


\subsection{Case $q=1$}

Since the diffusivity of the pipe's wall is small, although we get the same kind of the effective boundary condition (Robin), the heat transfer coefficient is lower than the original one.

Theorem 6.5. Let $q=1$ and let $\theta^{\varepsilon} \in H^{1}\left(P_{1+\varepsilon}\right)$ be the solution to the problem (2.11)-(2.13) and let (4.2) and (4.3) hold. Then

$$
\begin{aligned}
& \tilde{\theta}^{\varepsilon} \rightarrow \tilde{\theta} \text { weak } * \text { in } L^{\infty}\left(\tilde{P}_{1}\right), \\
& \tilde{\theta}^{\varepsilon} \rightarrow \tilde{\theta} \text { weakly in } H^{1}\left(\tilde{P}_{1}\right), \\
& \theta^{\varepsilon} \rightarrow \Theta 2 \mathrm{~s}, \\
& \varepsilon \nabla \theta^{\varepsilon} \rightarrow \frac{\partial \Theta}{\partial \rho} \mathbf{e}_{r} 2 \mathrm{~s},
\end{aligned}
$$

where $\tilde{\theta} \in H^{1}\left(\tilde{P}_{1}\right) \cap L^{\infty}\left(\tilde{P}_{1}\right)$ is the unique solution to the problem

$$
\begin{array}{lr}
-\Delta \tilde{\theta}+\operatorname{Pe} \tilde{v} \frac{\partial \tilde{\theta}}{\partial z}=0 & \text { in } \tilde{P}_{1}, \\
-\frac{\partial \tilde{\theta}}{\partial z}+\operatorname{Pe} \tilde{v} \tilde{\theta}=N_{z}^{f}\left(\tilde{F}_{\ell}-\tilde{\theta}\right) & \text { on } \tilde{\gamma}_{1}^{\ell}, \\
\frac{\partial \tilde{\theta}}{\partial z}-\operatorname{Pe} \tilde{v} \tilde{\theta}=N_{z}^{f}\left(\tilde{F}_{r}-\tilde{\theta}\right) & \text { on } \tilde{\gamma}_{1}^{r}, \\
\frac{\partial \tilde{\theta}}{\partial r}=\frac{\kappa N_{r}}{\kappa+N_{r}}(G-\tilde{\theta}) & \text { on } \tilde{S}_{1} .
\end{array}
$$

Proof. Again, the only difference is in the treatment of the integrals over $\tilde{P}_{\varepsilon}$ and $\tilde{S}_{1+\varepsilon}$. Since for $q=1$ from (4.24) we have

$$
\varepsilon\left|\nabla \theta^{\varepsilon}\right|_{L^{2}\left(P_{\varepsilon}\right)} \leq C \sqrt{\varepsilon}
$$

the assertion (c) from Theorem 5.2 applies and gives (6.29). Now we need an appropriate test function. Let $\Phi$ be a smooth function defined on

$$
P_{2}=\left\{(\rho, \varphi, z) \in \mathbb{R}^{3}: \rho \in\langle 0,2\rangle, \varphi \in\langle 0,2 \pi\rangle, z \in\langle 0, L\rangle\right\}
$$

and $\Phi=\tilde{\Phi} \circ F$. We define

$$
\Psi_{\varepsilon}(r, \varphi, z)= \begin{cases}\Phi(r, \varphi, z) & \text { in } P_{1} \\ \Phi\left(1+\frac{r-1}{\varepsilon}, \varphi, z\right) & \text { in } P_{\varepsilon}\end{cases}
$$

Then we have using the two-scale convergence (6.29)

$$
\begin{aligned}
& \varepsilon \int_{P_{\varepsilon}}\left(r \frac{\partial \theta^{\varepsilon}}{\partial r} \frac{\partial \Psi_{\varepsilon}}{\partial r}+\frac{1}{r} \frac{\partial \theta^{\varepsilon}}{\partial \varphi} \frac{\partial \Psi_{\varepsilon}}{\partial \varphi}+r \frac{\partial \theta^{\varepsilon}}{\partial z} \frac{\partial \Psi_{\varepsilon}}{\partial z}\right) \mathrm{d} r \mathrm{~d} \varphi \mathrm{d} z \\
& \quad=\frac{1}{\varepsilon} \int_{P_{\varepsilon}} \varepsilon \frac{\partial \theta^{\varepsilon}}{\partial r}(r, \varphi, z) \frac{\partial \Phi}{\partial \rho}\left(1+\frac{r-1}{\varepsilon}, \varphi, z\right) \mathrm{d} r \mathrm{~d} \varphi \mathrm{d} z+O(\varepsilon) \\
& \quad \rightarrow \int_{P_{2-1}} \frac{\partial \Theta}{\partial \rho}(\rho, \varphi, z) \frac{\partial \Phi}{\partial \rho}(\rho, \varphi, z) \mathrm{d} \rho \mathrm{d} \varphi \mathrm{d} z .
\end{aligned}
$$

Another integral that needs additional attention is

$$
\int_{\tilde{S}_{1+\varepsilon}} \tilde{\theta}^{\varepsilon} \tilde{\Psi}_{\varepsilon} \mathrm{d} a=(1+\varepsilon) \int_{S} \theta^{\varepsilon}(1+\varepsilon, \varphi, z) \Phi(2, \varphi, z) \mathrm{d} \varphi \mathrm{d} z
$$




$$
\begin{aligned}
= & \int_{1}^{1+\varepsilon} \int_{0}^{2 \pi} \int_{0}^{L} \frac{\partial \theta^{\varepsilon}}{\partial r}(r, \varphi, z) \Phi(2, \varphi, z) \mathrm{d} r \mathrm{~d} \varphi \mathrm{d} z \\
& +\int_{0}^{2 \pi} \int_{0}^{L} \theta^{\varepsilon}(1, \varphi, z) \Phi(2, \varphi, z) \mathrm{d} \varphi \mathrm{d} z .
\end{aligned}
$$

For the second integral from (6.1) and (6.3) and the trace theorem, we obviously have

$$
\int_{0}^{2 \pi} \int_{0}^{L} \theta^{\varepsilon}(1, \varphi, z) \Phi(2, \varphi, z) \mathrm{d} \varphi \mathrm{d} z \rightarrow \int_{0}^{2 \pi} \int_{0}^{L} \theta(1, \varphi, z) \Phi(2, \varphi, z) \mathrm{d} \varphi \mathrm{d} z .
$$

For the first term we apply the two-scale convergence (6.29) and obtain

$$
\frac{1}{\varepsilon} \int_{P_{\varepsilon}} \varepsilon \frac{\partial \theta^{\varepsilon}}{\partial r}(r, \varphi, z) \Phi(2, \varphi, z) r \mathrm{~d} r \mathrm{~d} \varphi \mathrm{d} z \rightarrow \int_{P_{2-1}} \frac{\partial \Theta}{\partial \rho}(\rho, \varphi, z) \Phi(2, \varphi, z) \mathrm{d} \rho \mathrm{d} \varphi \mathrm{d} z .
$$

Newton-Leibniz theorem and continuity of temperature at the contact, i.e. $\left.\Theta\right|_{r=1}=\left.\theta\right|_{r=1}$, now implies that

$$
\int_{\tilde{S}_{1+\varepsilon}} \tilde{\theta}^{\varepsilon} \tilde{\Psi}_{\varepsilon} \mathrm{d} a \rightarrow \int_{S} \Theta(2, \varphi, z) \Phi(2, \varphi, z) \mathrm{d} \varphi \mathrm{d} z=\frac{1}{2} \int_{\tilde{S}_{2}} \tilde{\Theta} \Phi \mathrm{d} a .
$$

Thus the two-scale problem reads

$$
\begin{aligned}
\int_{\tilde{P}_{1}} & \nabla \tilde{\theta} \cdot \nabla \tilde{\Phi} \mathrm{d} V-\operatorname{Pe} \int_{\tilde{P}_{1}} \tilde{v} \tilde{\theta} \frac{\partial \tilde{\Phi}}{\partial z} \mathrm{~d} V+\kappa \int_{P_{2-1}} \frac{\partial \Theta}{\partial \rho} \frac{\partial \Phi}{\partial \rho} \mathrm{d} \rho \mathrm{d} \varphi \mathrm{d} z \\
& +N_{z}^{f} \int_{\tilde{\gamma}_{1}^{\ell}} \tilde{\theta} \tilde{\Phi} \mathrm{d} a+N_{z}^{f} \int_{\tilde{\gamma}_{1}^{r}} \tilde{\theta} \tilde{\Phi} \mathrm{d} a+\frac{1}{2} N_{r} \int_{\tilde{S}_{2}} \tilde{\Theta} \tilde{\Phi} \mathrm{d} a \\
= & N_{z}^{f} \int_{\tilde{\gamma}_{1}^{\ell}} \tilde{F}_{\ell} \tilde{\Phi} \mathrm{d} a+N_{z}^{f} \int_{\tilde{\gamma}_{1}^{r}} \tilde{F}_{r} \tilde{\Phi} \mathrm{d} a+\frac{1}{2} N_{r} \int_{\tilde{S}_{2}} \tilde{G} \tilde{\Phi} \mathrm{d} a .
\end{aligned}
$$

Choosing $\Phi$ to be compactly supported in $\overline{P_{2-1}}$, i.e. equal zero for $0<r<1$, we arrive at

$$
\begin{aligned}
& \kappa \int_{P_{2-1}} \frac{\partial \Theta}{\partial \rho} \frac{\partial \Phi}{\partial \rho} \mathrm{d} \rho \mathrm{d} \varphi \mathrm{d} z+N_{r} \int_{S} \Theta(2, \varphi, z) \Phi(2, \varphi, z) \mathrm{d} \varphi \mathrm{d} z \\
& \quad=N_{r} \int_{S} G(\varphi, z) \Phi(2, \varphi, z) \mathrm{d} \varphi \mathrm{d} z .
\end{aligned}
$$

Thus

$$
\frac{\partial^{2} \Theta}{\partial \rho^{2}}=0 \text { for } 1<\rho<2, \quad \kappa \frac{\partial \Theta}{\partial \rho}=N_{r}(G-\Theta) \text { for } \rho=2 .
$$

Furthermore continuity of the temperature implies

$$
\Theta(1, \varphi, z)=\theta(1, \varphi, z)
$$

By simple integration we get

$$
\Theta(\rho, \varphi, z)=A(\varphi, z) \rho+B(\varphi, z)
$$

and then the boundary conditions give

$$
\kappa A=N_{r}(G-2 A-B)=0 \& A+B=\theta(1, \varphi, z)
$$

which implies

$$
A=\frac{N_{r}}{\kappa+N_{r}}(G-\theta(1, \varphi, z))
$$


Now we go back to (6.33) and take the test function $\Phi$ such that $\Phi(2, \varphi, z)=0$. Since now

$$
\begin{aligned}
\kappa \int_{P_{2-1}} \frac{\partial \Theta}{\partial \rho} \frac{\partial \Phi}{\partial \rho} \mathrm{d} \rho \mathrm{d} \varphi \mathrm{d} z & =\kappa \int_{P_{2-1}} A(\varphi, z) \frac{\partial \Phi}{\partial \rho}(\rho, \varphi, z) \mathrm{d} \rho \mathrm{d} \varphi \mathrm{d} z \\
& =-\kappa \int_{S} A(\varphi, z) \Phi(1, \varphi, z) \mathrm{d} \rho \mathrm{d} \varphi \mathrm{d} z
\end{aligned}
$$

we get the model: find $\tilde{\theta} \in H^{1}\left(\tilde{P}_{1}\right)$ such that

$$
\begin{gathered}
\int_{\tilde{P}_{1}} \nabla \tilde{\theta} \cdot \nabla \tilde{\Phi} \mathrm{d} V-\operatorname{Pe} \int_{\tilde{P}_{1}} \tilde{v} \tilde{\theta} \frac{\partial \tilde{\Phi}}{\partial z} \mathrm{~d} V+N_{z}^{f} \int_{\tilde{\gamma}_{1}^{\ell}} \tilde{\theta} \tilde{\Phi} \mathrm{d} a+N_{z}^{f} \int_{\tilde{\gamma}_{1}^{r}} \tilde{\theta} \tilde{\Phi} \mathrm{d} a \\
=N_{z}^{f} \int_{\tilde{\gamma}_{1}^{\ell}} \tilde{F}_{\ell} \tilde{\Phi} \mathrm{d} a+N_{z}^{f} \int_{\tilde{\gamma}_{1}^{r}} \tilde{F}_{r} \tilde{\Phi} \mathrm{d} a+\frac{\kappa N_{r}}{\kappa+N_{r}} \int_{\tilde{S}_{1}}(\tilde{G}-\tilde{\theta}) \tilde{\Phi} \mathrm{d} a,
\end{gathered}
$$

for all $\tilde{\Phi} \in H^{1}\left(\tilde{P}_{1}\right)$.

The uniqueness of solution of (6.34) is deduced as in the previous cases. Finally, we conclude that the differential form of our problem now reads (6.30) and (6.31).

\subsection{Case $q>1$}

In the last case the diffusivity of the pipe's wall is very small so that it acts as an isolator. There is no heat exchange between the fluid and the exterior medium. Thus the effective boundary condition is the homogeneous Neumann boundary condition.

Theorem 6.6. Let $q>1$ and let $\tilde{\theta}^{\varepsilon} \in H^{1}\left(\tilde{P}_{1+\varepsilon}\right)$ be a weak solution to the problem (2.11)-(2.13). Assume that (4.2) and (4.3) hold. Then

$$
\begin{array}{lll}
\tilde{\theta}^{\varepsilon} \rightarrow \tilde{\theta} & \text { weak } * \text { in } & L^{\infty}\left(\tilde{P}_{1}\right), \\
\tilde{\theta}^{\varepsilon} \rightarrow \tilde{\theta} & \text { weakly in } & H^{1}\left(\tilde{P}_{1}\right),
\end{array}
$$

where $\tilde{\theta} \in H^{1}\left(\tilde{P}_{1}\right) \cap L^{\infty}\left(\tilde{P}_{1}\right)$ is the unique solution to the problem

$$
\begin{array}{lr}
-\Delta \tilde{\theta}+\operatorname{Pe} \tilde{v} \frac{\partial \tilde{\theta}}{\partial z}=0 & \text { in } \tilde{P}_{1}, \\
-\frac{\partial \tilde{\theta}}{\partial z}+\operatorname{Pe} \tilde{v} \tilde{\theta}=N_{z}^{f}\left(\tilde{F}_{\ell}-\tilde{\theta}\right) & \text { on } \tilde{\gamma}_{1}^{\ell}, \\
\frac{\partial \tilde{\theta}}{\partial z}-\operatorname{Pe} \tilde{v} \tilde{\theta}=N_{z}^{f}\left(\tilde{F}_{r}-\tilde{\theta}\right) & \text { on } \tilde{\gamma}_{1}^{r} \\
\frac{\partial \tilde{\theta}}{\partial r}=0 & \text { on } \tilde{S}_{1} .
\end{array}
$$

Proof. We proceed as in the case $q=1$ and take the test function $\Psi_{\varepsilon}$ as in (6.32). However now we have

$$
\varepsilon^{q} \int_{P_{\varepsilon}}\left(r \frac{\partial \theta^{\varepsilon}}{\partial r} \frac{\partial \Psi_{\varepsilon}}{\partial r}+\frac{1}{r} \frac{\partial \theta^{\varepsilon}}{\partial \varphi} \frac{\partial \Psi_{\varepsilon}}{\partial \varphi}+r \frac{\partial \theta^{\varepsilon}}{\partial z} \frac{\partial \Psi_{\varepsilon}}{\partial z}\right) \mathrm{d} r \mathrm{~d} \varphi \mathrm{d} z \rightarrow 0
$$

On the other hand, due to the a priori estimate (4.20), we have

$$
\int_{S}\left|\theta^{\varepsilon}(1+\varepsilon, \varphi, z)\right|^{2} \mathrm{~d} \varphi \mathrm{d} z<C
$$


so that

$$
\omega_{\varepsilon}(\varphi, z)=\theta^{\varepsilon}(1+\varepsilon, \varphi, z)
$$

is bounded in $\mathcal{H}$. Thus, extracting the subsequence if necessary, we have

$$
\omega_{\varepsilon} \rightarrow \omega \text { weakly in } \mathcal{H}
$$

for some $\omega \in \mathcal{H}$. Now

$$
(1+\varepsilon) \int_{S} \theta^{\varepsilon}(1+\varepsilon, \varphi, z) \Phi(2, \varphi, z) \mathrm{d} \varphi \mathrm{d} z \rightarrow \int_{S} \omega(\varphi, z) \Phi(2, \varphi, z) \mathrm{d} \varphi .
$$

Consequently the limit problem reads

$$
\begin{aligned}
\int_{\tilde{P}_{1}} & \nabla \tilde{\theta} \cdot \nabla \tilde{\Phi} \mathrm{d} V-\operatorname{Pe} \int_{\tilde{P}_{1}} \tilde{v} \tilde{\theta} \frac{\partial \tilde{\Phi}}{\partial z} \mathrm{~d} V \\
& +N_{z}^{f} \int_{\tilde{\gamma}_{1}^{\ell}} \tilde{\theta} \tilde{\Phi} \mathrm{d} a+N_{z}^{f} \int_{\tilde{\gamma}_{1}^{r}} \tilde{\theta} \tilde{\Phi} \mathrm{d} a+N_{r} \int_{S_{2}} \omega \Phi \mathrm{d} a \\
= & N_{z}^{f} \int_{\tilde{\gamma}_{1}^{\ell}} \tilde{F}_{\ell} \tilde{\Phi} \mathrm{d} a+N_{z}^{f} \int_{\tilde{\gamma}_{1}^{r}} \tilde{F}_{r} \tilde{\Phi} \mathrm{d} a+N_{r} \int_{S_{2}} G \Phi \mathrm{d} a .
\end{aligned}
$$

Choosing again the test function $\Phi$ to be compactly supported in $\overline{P_{2-1}}$ (i.e. equal zero for $0<r<1$ ) we get $\omega=G$ and therefore the model is given by: find $\tilde{\theta} \in H^{1}\left(\tilde{P}_{1}\right)$ such that

$$
\begin{aligned}
& \int_{\tilde{P}_{1}} \nabla \tilde{\theta} \cdot \nabla \tilde{\Phi} \mathrm{d} V-\operatorname{Pe} \int_{\tilde{P}_{1}} \tilde{v} \tilde{\theta} \frac{\partial \tilde{\Phi}}{\partial z} \mathrm{~d} V+N_{z}^{f} \int_{\tilde{\gamma}_{1}^{\ell}} \tilde{\theta} \tilde{\Phi} \mathrm{d} a+N_{z}^{f} \int_{\tilde{\gamma}_{1}^{r}} \tilde{\theta} \tilde{\Phi} \mathrm{d} a \\
& \quad=N_{z}^{f} \int_{\tilde{\gamma}_{1}^{\ell}} \tilde{F}_{\ell} \tilde{\Phi} \mathrm{d} a+N_{z}^{f} \int_{\tilde{\gamma}_{1}^{r}} \tilde{F}_{r} \tilde{\Phi} \mathrm{d} a
\end{aligned}
$$

for all $\tilde{\Phi} \in H^{1}\left(\tilde{P}_{1}\right)$. This implies (6.37) and (6.38). Uniqueness of the solution of (6.40) implies the convergence of the whole families as before.

\section{UNIFIED MODEL WiTh THE SAME ASYMPTOTICS IN EACH OF THE CASES}

The original $\varepsilon$ problem from which we start the asymptotic analysis is done on a fluid domain $\tilde{P}_{1}$ and on the pipe's wall $\tilde{P}_{\varepsilon}$. In the asymptotic analysis we obtained five different effective models which are formulated on only on the fluid domain with a difference in the boundary condition on the interface between the pipe and the wall. In a real life situation one is left to choose either to use $\varepsilon$ problem or to decide which of the effective models to use. The drawback of using $\varepsilon$ model is twofold, its mathematical structure is more complicated and the small thickness of the pipe's wall in simulations implies that regular triangulation of the pipe's wall is very large leading to time consuming computations. On the other hand usually it is not completely clear how to read from the real data which effective model to use. Thus in this section we formulate unified model given on $\tilde{P}_{1}$ which has the same asymptotics as the original problem (2.11)-(2.13). The model we formulate in (7.1) is given on the fluid domain $\tilde{P}_{1}$ only and thus is of numerical complexity of the limit models, however its asymptotic limits are the same as of the initial $\varepsilon$ problem. The model is given for two unknown functions, $\tilde{\theta}(\varepsilon)$ which is the temperature in the pipe and belongs to

$$
\mathcal{V}_{-1}=\left\{\tilde{\psi} \in H^{1}\left(\tilde{P}_{1}\right): \tilde{\psi} \circ F \in H^{1}(S)\right\}
$$


and $\tilde{\theta}_{0}(\varepsilon) \in L^{2}\left(\tilde{S}_{1}\right)$ the function that can be considered as the temperature at the outer (and sometimes inner) pipe's wall. The model reads: find $\left(\tilde{\theta}(\varepsilon), \tilde{\theta}_{0}(\varepsilon)\right) \in \mathcal{V}_{-1} \times L^{2}\left(\tilde{S}_{1}\right)$ such that

$$
\begin{aligned}
\int_{\tilde{P}_{1}} & \nabla \tilde{\theta}(\varepsilon) \cdot \nabla \tilde{\psi} \mathrm{d} V-\operatorname{Pe} \int_{\tilde{P}_{1}} \tilde{v} \tilde{\theta}(\varepsilon) \frac{\partial \tilde{\psi}}{\partial z} \mathrm{~d} V \\
& +\varepsilon^{q+1} \kappa \int_{\tilde{S}_{1}} \nabla_{\tau} \tilde{\theta}(\varepsilon) \cdot \nabla_{\tau} \tilde{\psi} \mathrm{d} a+\varepsilon^{q-1} \kappa \int_{\tilde{S}_{1}}\left(\tilde{\theta}_{0}(\varepsilon)-\tilde{\theta}(\varepsilon)\right)\left(\tilde{\psi}_{0}-\tilde{\psi}\right) \mathrm{d} a \\
& +N_{r}(1+\varepsilon) \int_{\tilde{S}_{1}} \tilde{\theta}_{0}(\varepsilon) \tilde{\psi}_{0} \mathrm{~d} a+N_{z}^{f} \int_{\tilde{\gamma}_{1}^{e}} \tilde{\theta}(\varepsilon) \tilde{\psi} \mathrm{d} a+N_{z}^{f} \int_{\tilde{\gamma}_{1}^{r}} \tilde{\theta}(\varepsilon) \tilde{\psi} \mathrm{d} a \\
= & N_{r}(1+\varepsilon) \int_{\tilde{S}_{1}} \tilde{G} \tilde{\psi}_{0} \mathrm{~d} a+N_{z}^{f} \int_{\tilde{\gamma}_{1}^{e}} \tilde{F}_{l} \tilde{\psi} \mathrm{d} a+N_{z}^{f} \int_{\tilde{\gamma}_{1}^{r}} \tilde{F}_{r} \tilde{\psi} \mathrm{d} a,
\end{aligned}
$$

for all $\left(\tilde{\psi}, \tilde{\psi}_{0}\right) \in \mathcal{V}_{-1} \times L^{2}\left(\tilde{S}_{1}\right)$.

Note that the term with $\varepsilon^{q-1}$ is an approximation of the term $\partial_{r} \tilde{\theta} \partial_{r} \tilde{\psi}$ with $\tilde{\theta}_{0}$ playing the role of temperature at outer side of the pipe's wall. Note also that the term $(1+\varepsilon)$ multiplies the coefficient $N_{r}$ as in the original problem (2.14) in order for this approximation model to be closer to the original problem. For limits this factor is irrelevant.

Lemma 7.1 (A priori estimates for the unified model). Let $\left(\tilde{\theta}(\varepsilon), \tilde{\theta}_{0}(\varepsilon)\right) \in \mathcal{V}_{-1} \times L^{2}\left(\tilde{S}_{1}\right)$ be the solution of (7.1). Suppose that (4.2) and (4.3) hold. Then for all $q \in \mathbb{R}$ the families

$$
\begin{aligned}
& |\tilde{\theta}(\varepsilon)|_{H^{1}\left(\tilde{P}_{1}\right)}, \quad\left|\tilde{\theta}_{0}(\varepsilon)^{2}\right|_{L^{2}\left(\tilde{\gamma}_{1}^{\ell}\right)}, \quad\left|\tilde{\theta}_{0}(\varepsilon)^{2}\right|_{L^{2}\left(\tilde{\gamma}_{1}^{r}\right)}, \\
& \left|\tilde{\theta}_{0}(\varepsilon)^{2}\right|_{L^{2}\left(\tilde{S}_{1}\right)}, \quad \varepsilon^{\frac{q+1}{2}}\left|\nabla_{\tau} \tilde{\theta}(\varepsilon)\right|_{L^{2}\left(\tilde{S}_{1}\right)}, \quad \varepsilon^{\frac{q-1}{2}}\left|\tilde{\theta}(\varepsilon)-\tilde{\theta}_{0}(\varepsilon)\right|_{L^{2}\left(\tilde{S}_{1}\right)}, \quad\left|\tilde{\theta}_{0}(\varepsilon)\right|_{L^{2}\left(\tilde{S}_{1}\right)}
\end{aligned}
$$

are uniformly bounded with respect to $\varepsilon \in\langle 0,1\rangle$.

Proof. The main obstacle in the proof is the term with Pe. However applying the same technique as in the proof of Theorem 4.1 the statement of the lemma follows.

Theorem 7.2. Limits of the model given by (7.1) when $\varepsilon$ tends to zero in the same regimes are the same as in the case of (4.1).

Proof. From the a priori estimates we obtain that there exist $\tilde{\theta} \in H^{1}\left(\tilde{P}_{1}\right), \tilde{\theta}_{0}, \tilde{\theta}_{r}, \tilde{\theta}_{\tau} \in L^{2}\left(\tilde{S}_{1}\right)$, such that the following weak convergences (at a subsequence) hold

$$
\begin{aligned}
\tilde{\theta}(\varepsilon) \rightarrow \tilde{\theta} & \text { in } H^{1}\left(\tilde{P}_{1}\right), \\
\tilde{\theta}_{0}(\varepsilon)-\tilde{\theta}_{0} & \text { in } L^{2}\left(\tilde{S}_{1}\right), \\
\varepsilon^{\frac{q-1}{2}}\left(\tilde{\theta}(\varepsilon)-\tilde{\theta}_{0}(\varepsilon)\right)-\tilde{\theta}_{r} & \text { in } L^{2}\left(\tilde{S}_{1}\right), \\
\varepsilon^{\frac{q+1}{2}} \nabla_{\tau} \tilde{\theta}(\varepsilon)-\tilde{\theta}_{\tau} & \text { in } L^{2}\left(\tilde{S}_{1}\right), \\
\tilde{\theta}(\varepsilon)-\tilde{\theta} & \text { in } L^{2}\left(\tilde{S}_{1}\right), \\
\tilde{\theta}(\varepsilon) \rightarrow \tilde{\theta} & \text { in } L^{2}\left(\tilde{\gamma}_{1}^{\ell}\right), \\
\tilde{\theta}(\varepsilon) \rightarrow \tilde{\theta} & \text { in } L^{2}\left(\tilde{\gamma}_{1}^{r}\right) .
\end{aligned}
$$

For $q<-1$ the convergences $(7.2)$ imply that $\tilde{\theta}$ is a constant on $\tilde{S}_{1}$ and that $\tilde{\theta}_{0}=\left.\tilde{\theta}\right|_{\tilde{S}_{1}}$. Thus $\tilde{\theta} \in \mathcal{V}_{\langle-\infty,-1\rangle}$. Then for $\tilde{\psi} \in \mathcal{V}_{\langle-\infty,-1\rangle}$ and the constant $\tilde{\psi}_{0}=\left.\tilde{\psi}\right|_{\tilde{S}_{1}}$ in the limit of (7.1) we obtain the model (6.17).

For $q=-1$ the convergences $(7.2)$ imply that $\tilde{\theta} \in \mathcal{V}_{-1}$ and that $\tilde{\theta}_{0}=\left.\tilde{\theta}\right|_{\tilde{S}_{1}}$. Then for $\tilde{\psi} \in \mathcal{V}_{-1}$ and $\tilde{\psi}_{0}=\left.\tilde{\psi}\right|_{\tilde{S}_{1}}$ in the limit of (7.1) we obtain the model (6.21). 
For $q \in\langle-1,1\rangle$ the convergences $(7.2)$ imply that $\tilde{\theta} \in H^{1}\left(\tilde{P}_{1}\right)$ and that $\tilde{\theta}_{0}=\left.\tilde{\theta}\right|_{\tilde{S}_{1}}$. Then for $\tilde{\psi} \in \mathcal{V}_{-1}$ and $\tilde{\psi}_{0}=\left.\tilde{\psi}\right|_{\tilde{S}_{1}}$ in the limit of (7.1) we obtain the model $(6.25)$.

For $q=1$ the convergences $(7.2)$ imply that $\tilde{\theta} \in H^{1}\left(\tilde{P}_{1}\right)$ and that $\tilde{\theta}_{0}(\varepsilon) \rightarrow \tilde{\theta}_{0} \in L^{2}\left(\tilde{S}_{1}\right)$. Then for $\tilde{\psi} \in \mathcal{V}_{-1}$ in the limit of (7.1) we obtain the equation

$$
\begin{aligned}
\int_{\tilde{P}_{1}} & \nabla \tilde{\theta} \cdot \nabla \tilde{\psi} \mathrm{d} V-\operatorname{Pe} \int_{\tilde{P}_{1}} \tilde{v} \tilde{\theta} \frac{\partial \tilde{\psi}}{\partial z} \mathrm{~d} V+\kappa \int_{\tilde{S}_{1}}\left(\tilde{\theta}_{0}-\tilde{\theta}\right)\left(\tilde{\psi}_{0}-\tilde{\psi}\right) \mathrm{d} a \\
& +N_{r} \int_{\tilde{S}_{1}} \tilde{\theta}_{0} \tilde{\psi}_{0} \mathrm{~d} a+N_{z}^{f} \int_{\tilde{\gamma}_{1}^{e}} \tilde{\theta} \tilde{\psi} \mathrm{d} a+N_{z}^{f} \int_{\tilde{\gamma}_{1}^{r}} \tilde{\theta} \tilde{\psi} \mathrm{d} a \\
= & N_{r} \int_{\tilde{S}_{1}} \tilde{G} \tilde{\psi}_{0} \mathrm{~d} a+N_{z}^{f} \int_{\tilde{\gamma}_{1}^{e}} \tilde{F}_{l} \tilde{\psi} \mathrm{d} a+N_{z}^{f} \int_{\tilde{\gamma}_{1}^{r}} \tilde{F}_{r} \tilde{\psi} \mathrm{d} a .
\end{aligned}
$$

From the equation for the test function $\tilde{\psi}_{0}$ we obtain the expression for $\tilde{\theta}_{0}$. Namely

$$
\kappa \int_{\tilde{S}_{1}}\left(\tilde{\theta}_{0}-\tilde{\theta}\right) \tilde{\psi}_{0} \mathrm{~d} a+N_{r} \int_{\tilde{S}_{1}} \tilde{\theta}_{0} \tilde{\psi}_{0} \mathrm{~d} a=N_{r} \int_{\tilde{S}_{1}} \tilde{G} \tilde{\psi}_{0} \mathrm{~d} a
$$

implies that $\left(\kappa+N_{r}\right) \tilde{\theta}_{0}-\kappa \tilde{\theta}=N_{r} \tilde{G}$, i.e.

$$
\tilde{\theta}_{0}=\frac{\kappa}{\kappa+N_{r}} \tilde{\theta}+\frac{N_{r}}{\kappa+N_{r}} \tilde{G}
$$

Now we insert this in (7.3) for the test function $\tilde{\psi}$ and obtain the model (6.34).

Finally in the case $q>1$, the convergences (7.2) imply that $\tilde{\theta} \in H^{1}\left(\tilde{P}_{1}\right)$ and $\tilde{\theta}_{0} \in L^{2}\left(\tilde{S}_{1}\right)$ and that in the limit we obtain

$$
\begin{aligned}
\int_{\tilde{P}_{1}} & \nabla \tilde{\theta} \cdot \nabla \tilde{\psi} \mathrm{d} V-\operatorname{Pe} \int_{\tilde{P}_{1}} \tilde{v} \tilde{\theta} \frac{\partial \tilde{\psi}}{\partial z} \mathrm{~d} V \\
& +N_{r} \int_{\tilde{S}_{1}} \tilde{\theta}_{0} \tilde{\psi}_{0} \mathrm{~d} a+N_{z}^{f} \int_{\tilde{\gamma}_{1}^{e}} \tilde{\theta} \tilde{\psi} \mathrm{d} a+N_{z}^{f} \int_{\tilde{\gamma}_{1}^{r}} \tilde{\theta} \tilde{\psi} \mathrm{d} a \\
= & N_{r} \int_{\tilde{S}_{1}} \tilde{G} \tilde{\psi}_{0} \mathrm{~d} a+N_{z}^{f} \int_{\tilde{\gamma}_{1}^{e}} \tilde{F}_{l} \tilde{\psi} \mathrm{d} a+N_{z}^{f} \int_{\tilde{\gamma}_{1}^{r}} \tilde{F}_{r} \tilde{\psi} \mathrm{d} a .
\end{aligned}
$$

From the equation for the test function $\tilde{\psi}_{0}$ we obtain that $\tilde{\theta}_{0}=\tilde{G}$ and then the equation (7.4) is exactly equal to $(6.40)$.

Uniqueness of solutions of the all limit problems implies that actually all $\varepsilon$ families are convergent.

\section{NumERICAL EXAMPLES}

In this section we present numerical simulations of the original $\varepsilon$ problem (4.1) and the unified model (7.1) in five different regimes, in particular for the values of parameter $q \in\{-5,-1,0,1,5\}$ to illustrate different behavior of the solution in different regimes. Moreover, we compare the results of both models together with five limit models to get an idea if the model (7.1) is a good approximation of the original problem. In all computations all parameters, besides of the parameter $q$, are the same and given in Table 1 . We present computations only for $\varepsilon=1 / 8$ which corresponds to rather thick pipe's wall compared to the inner radius of the pipe since in this case the the error of the limit model is already small, less then $5 \%$. For smaller $\varepsilon$ the limit model and the unified model should provide only better approximation.

The computation is done in cylindrical coordinates but with assumed independence of all unknowns on the variable $\varphi$, so the solution can be represented by a two-variable function. This significantly reduces the size of the problem and still provides qualitative behavior of the solution. The mesh for the numerical approximation 
TABLE 1. The set of parameters in numerical examples.

\begin{tabular}{ll}
\hline \hline Parameter & Value \\
\hline$\varepsilon$ & $2^{-3}$ \\
$\kappa$ & 1 \\
$\mathrm{Pe}$ & 0 \\
$L$ & 1 \\
$R$ & 1 \\
$\alpha_{f}$ & 1 \\
$\alpha_{s}$ & $\kappa \varepsilon^{q}$ \\
$N_{r}$ & 1 \\
$N_{z}$ & 0 \\
$G$ & $(z-0.5)+1$ \\
\hline
\end{tabular}

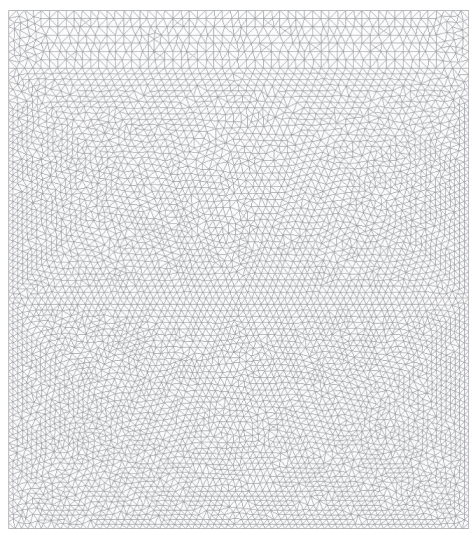

Figure 1. Mesh for the solution using FreeFem++.

TABLE 2. Minimal and maximal values of the temperature in each of the cases.

\begin{tabular}{lll}
\hline \hline$q$ & Min & Max \\
\hline-5 & 1 & 1 \\
-1 & 0.9647 & 1.035 \\
0 & 0.8825 & 1.117 \\
1 & 0.7361 & 1.264 \\
5 & 0.5 & 1.5 \\
\hline
\end{tabular}

of the solution of the original problem (4.1) using FreeFem++ (see [6]) is plotted in Figure 1. The thin layer of the pipe's wall can be clearly seen from the figure. For the unified model from Section 7 and the limit models we use the same mesh only on the fluid domain $[0,1]^{2}$.

To get more detailed description only half of the fluid domain is plotted, namely for $r \in[0.5,1+\varepsilon]$. In order to see only the effects of the difference in thermal conductivities of the fluid and the material of the pipe's wall we take the Peclet number as well as the Nusselt number $N_{z}^{f}$ to be zero.

In order to get a clear difference among solutions all plots are plotted on the same scale adjusted according to the case $q=-1$, but the minimal and maximal values of the solution in all of the cases are given in Table 2 . 


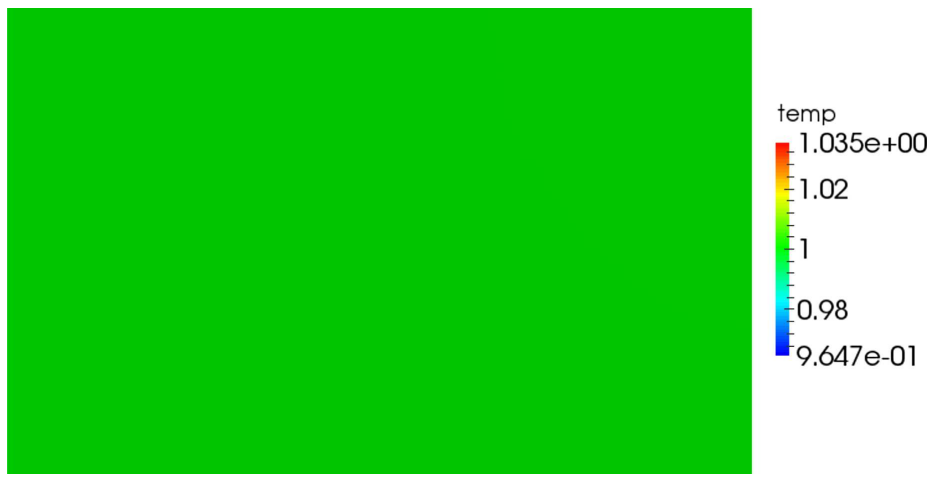

Figure 2. Solution for $q=-5$.

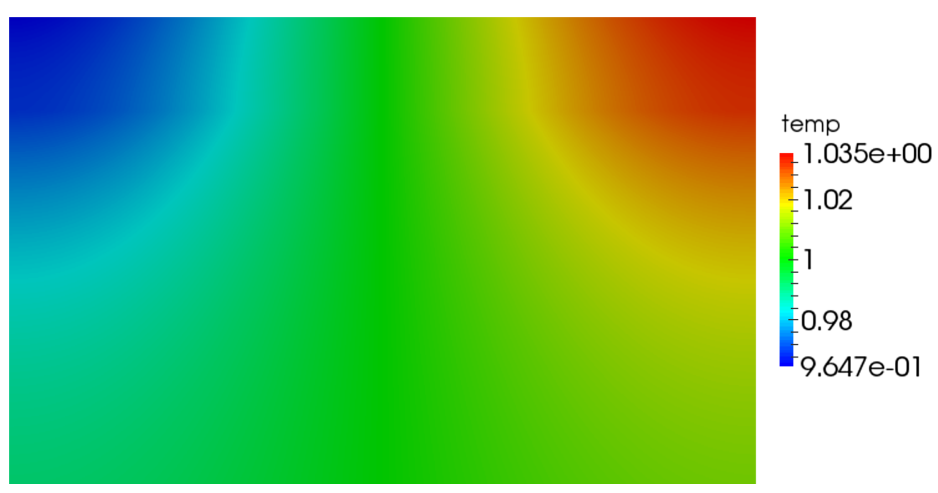

FiguRE 3. Solution for $q=-1$.

The solutions of the original problem (4.1) are given in Figures 2-6. In the Figure 2 the solution for $q=-5$ is plotted. In this case the thermal conductivity of the pipe's wall is so large that the temperature in the wall and in the pipe is constant. The constant is exactly equal to the mean value of the outer temperature $\bar{G}$ which, in this case, is equal to 1.

In Figure 3 the simulation for the value of parameter $q=-1$ is given. In this case the thermal conductivity is a bit smaller, but still large enough so the longitudinal conductivity is important. This leads to some difference in the maximal and minimal temperature but which is significantly smaller than in the classical engineering case $q \in\langle-1,1\rangle$ for which the simulation is given in Figure 4.

In Figure 5 the simulation for $q=1$ is given. The pipe's wall still conducts the heat significantly enough so it influences the temperature of the fluid, however the effective thermal coefficient is smaller. Finally in Figure 6 the thermal conduction is much smaller in the pipe's wall so the wall is effectively an insulator. There is largest difference in the temperature, which is exactly equal to the difference in the outer temperature $G$, but this difference is only in the pipe's wall, while in the fluid part the temperature is almost constant.

We also made numerical simulations for the unified model (7.1) and five limit models. However we skip the plots since they are qualitatively the same as the results of the original model (4.1), but we compare the differences between different approximations in Tables $3-5$.

In Table 3 the relative $H^{1}$ norm of differences of the numerical approximation of the original problem (4.1), the unified model (7.1) and the corresponding limit model, but only inside the pipe, i.e. in $[0,1]^{2}$, are presented for the same values of $q$. First note that all differences between the solution of the original problem (4.1) and the 


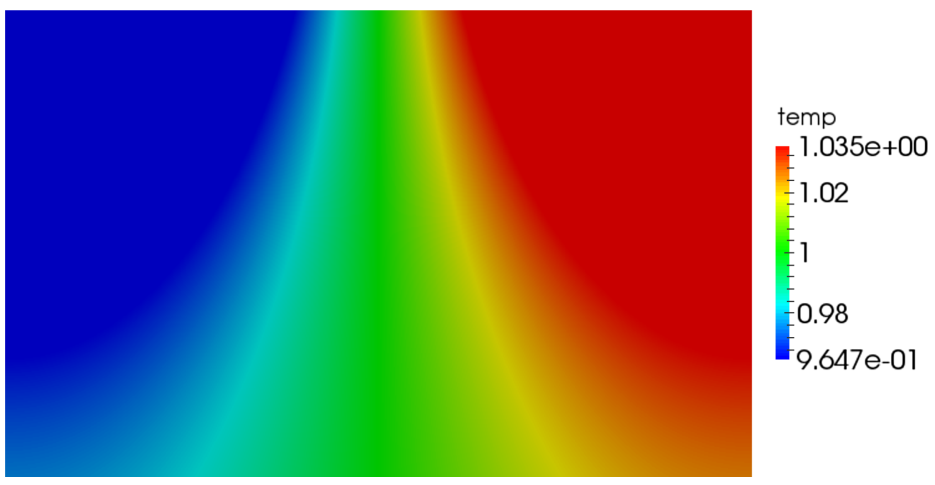

Figure 4. Solution for $q=0$.

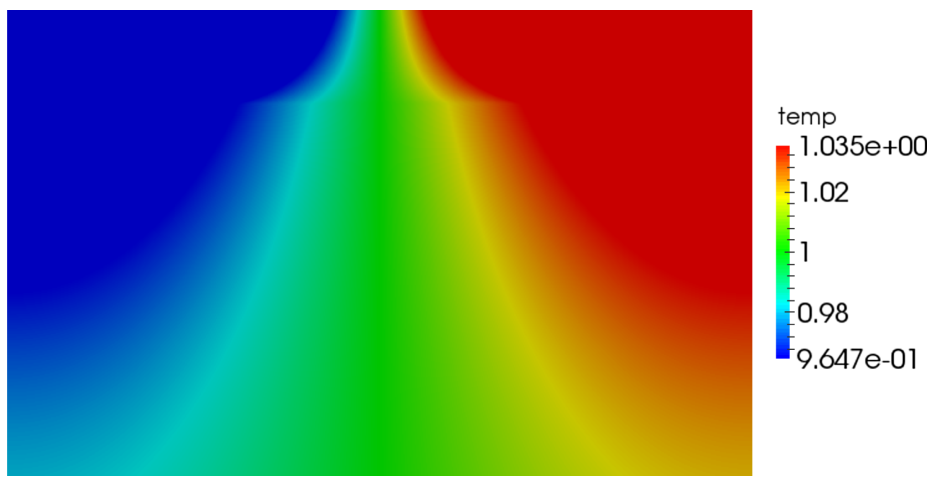

FiguRe 5. Solution for $q=1$.

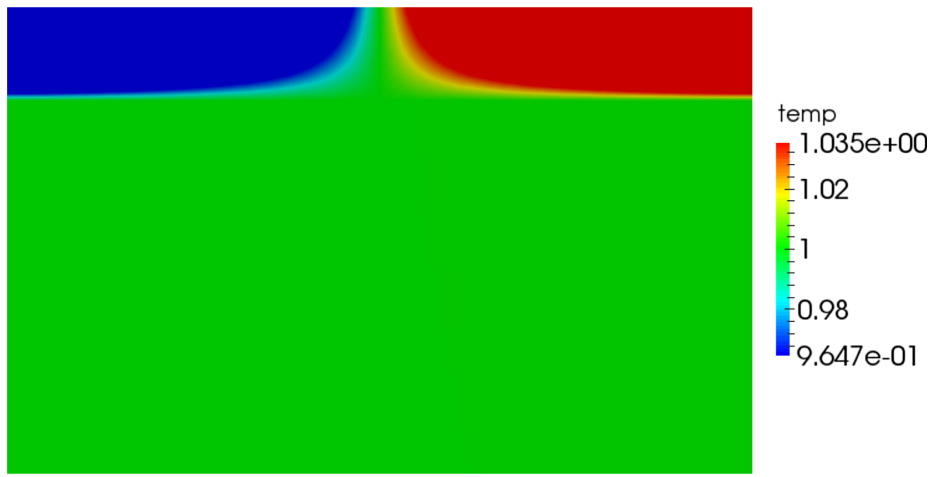

Figure 6. Solution for $q=5$. 
TABLE 3. Relative $H^{1}$ differences of the numerical approximation of the problem (4.1), unified model (7.1) and the corresponding limit model.

\begin{tabular}{llllll}
\hline \hline$H^{1}$ differences, $[0,1]^{2}$ & $q=-5$ & $q=-1$ & $q=0$ & $q=1$ & $q=5$ \\
\hline$(4.1)-(7.1)$ & $1.22 \times 10^{-6}$ & $2.97 \times 10^{-3}$ & $4.46 \times 10^{-3}$ & $1.63 \times 10^{-3}$ & $1.83 \times 10^{-6}$ \\
$(4.1)$-limit & $1.36 \times 10^{-5}$ & $9.04 \times 10^{-4}$ & $4.37 \times 10^{-2}$ & $2.13 \times 10^{-3}$ & $5.22 \times 10^{-5}$ \\
$(7.1)$-limit & $1.48 \times 10^{-5}$ & $3.83 \times 10^{-3}$ & $3.93 \times 10^{-2}$ & $5.10 \times 10^{-4}$ & $5.05 \times 10^{-5}$ \\
\hline
\end{tabular}

TABLE 4 . Relative $L^{\infty}$ differences of the numerical approximation of the problem (4.1), unified model (7.1) and the corresponding limit model.

\begin{tabular}{llllll}
\hline \hline$L^{\infty}$ differences, $[0,1]^{2}$ & $q=-5$ & $q=-1$ & $q=0$ & $q=1$ & $q=5$ \\
\hline (4.1)-(7.1) & $9.85 \times 10^{-7}$ & $2.37 \times 10^{-3}$ & $3.87 \times 10^{-3}$ & $1.38 \times 10^{-3}$ & $1.28 \times 10^{-6}$ \\
(4.1)-limit & $1.05 \times 10^{-5}$ & $5.97 \times 10^{-4}$ & $3.39 \times 10^{-2}$ & $1.80 \times 10^{-3}$ & $4.10 \times 10^{-5}$ \\
(7.1)-limit & $1.14 \times 10^{-5}$ & $2.89 \times 10^{-3}$ & $3.01 \times 10^{-2}$ & $4.24 \times 10^{-4}$ & $4.01 \times 10^{-5}$ \\
\hline
\end{tabular}

TABLE 5. Relative $L^{2}$ and $L^{\infty}$ differences of the numerical approximation of the original problem (4.1) and the unified model (7.1) in the pipe's wall (in $[0,1] \times[1,1+\varepsilon]$ ).

\begin{tabular}{llllll}
\hline \hline Differences in pipe's wall & $q=-5$ & $q=-1$ & $q=0$ & $q=1$ & $q=5$ \\
\hline$L^{2}$ differences in $[0,1] \times[1,1+\varepsilon]$ & $1.11 \times 10^{-6}$ & $2.97 \times 10^{-3}$ & $6.49 \times 10^{-3}$ & $6.50 \times 10^{-3}$ & $2.97 \times 10^{-3}$ \\
$L^{\infty}$ differences in [0,1] $\times[1,1+\varepsilon]$ & $2.23 \times 10^{-6}$ & $6.22 \times 10^{-3}$ & $1.75 \times 10^{-2}$ & $2.62 \times 10^{-2}$ & $1.33 \times 10^{-2}$ \\
$L^{2}$ differences on $[0,1] \times\{\varepsilon\}$ & $1.34 \times 10^{-6}$ & $3.68 \times 10^{-3}$ & $8.74 \times 10^{-3}$ & $9.81 \times 10^{-3}$ & $1.10 \times 10^{-5}$ \\
\hline
\end{tabular}

corresponding limit model are at most $4.5 \%$ which implies that the limit models are rather good approximation of the original problem. However, when we consider the approximation of the original problem (4.1) by the unified model (7.1) we see that the error is at most $0.46 \%$ which we find to be excellent. Also note that in all cases except $q=-1$ the approximation by the unified model (7.1) is better then by the corresponding limit model. Furthermore, in the case $q=-1$ the error of the unified model is less then $0.145 \%$.

Since the max temperature may be significant in Table 4 we present the relative $L^{\infty}$ differences as well. The behavior of the differences is quite similar to the observed one for the $H^{1}$ norm.

The unified model (and limit models as well) is given only on the domain of the pipe's interior. Thus to compare its solution with the solution of the original problem (4.1) in the pipe's wall we have to build an approximation in it. However in the case of the unified model (7.1) we have an additional unknown function $\tilde{\theta}_{0}(\varepsilon)$ which corresponds to the temperature at the outer boundary of the pipe's wall (as already noted $\left(\tilde{\theta}_{0}(\varepsilon)-\tilde{\theta}(\varepsilon)\right) / \varepsilon$ is an approximation for $\left.\partial_{r} \tilde{\theta}\right)$. Thus it is natural to build the affine approximation in the pipe's wall over the cross-section, i.e. the variable $r$. We compare this approximation with the solution of the original problem (4.1) in $L^{2}$ and $L^{\infty}$ norm in Table 5 .

In Table 5 we also compare the function $\tilde{\theta}_{0}(\varepsilon)$ with the solution of the original problem on the outer boundary of the pipe's wall. Having in mind that the chosen $\varepsilon=1 / 8$ is not small for the asymptotics we find the affine approximation excellent.

Acknowledgements. The authors are grateful to the anonymous referees for improving clarity and completeness of the paper. This work has been supported by the grant HRZZ IP-2018-01-2735 of the Croatian Science Foundation. 


\section{REFERENCES}

[1] N.S. Bakhvalov and G. Panasenko, Homogenisation: Averaging Processes in Periodic Media. Kluwer (1989).

[2] D. Caillerie, The effect of a thin inclusion of high rigidity in an elastic body. Math. Methods Appl. Sci. 2 (1980) 251-270.

[3] S.W. Churchill and H. Chu, Correlating equations for laminar and turbulent free convection from a vertical plate. Int. J. Heat Mass Trans. 18 (1975) 1323-1329.

[4] J. Droniou, Solving convection-diffusion equations with mixed, Neumann and Fourier boundary conditions and measures as data, by duality method. Adv. Differ. Equ. 5 (2000) 1341-1396.

[5] J. Fernandez-Seara, F.J. Uhia and J.A. Dopazo, Experimental transient natural convection heat transfer from a vertical cylindrical tank. Appl. Thermal Eng. 31 (2011) 1915-1922.

[6] F. Hecht, New development in FreeFem++. J. Numer. Math. 20 (2012) 251-266.

[7] J.P. Holman, Heat Transfer, 9th edition. Mc Graw Hill (2002).

[8] M. Ljulj and J. Tambača, 3D structure-2D plate interaction problem. Math. Mech. Solids 24 (2019) 3354-3377.

[9] S. Marušić and E. Marušić-Paloka, Two-scale convergence for thin domains and its applications to some lower-dimensional models in fluid mechanics. Asymptotic Anal. 23 (2000) 23-57.

[10] S. Marušić and E. Marušić-Paloka, Reduction of dimension for parabolic equations via two-scale convergence. In: Proceedings of ApplMat 99, Dubrovnik, 1999, Department of Mathematics, University of Zagreb (2001) 155-164.

[11] S. Marušić, E. Marušić-Paloka and I. Pažanin, Effects of strong convection on the cooling process for a long or thin pipe. C.R. Mec. 336 (2008) 493-499.

[12] E. Marušić-Paloka, Mathematical modeling of junctions in fluid mechanics via two-scale convergence. J. Math. Anal. App. 480 (2019) 123399.

[13] E. Marušić-Paloka and I. Pažanin, Non-isothermal fluid flow through a thin pipe with cooling. Appl. Anal. 88 (2009) $495-515$.

[14] E. Marušić-Paloka and I. Pažanin, Modelling of heat transfer in a laminar flow through a helical pipe. Math. Comput. Model. 50 (2009) 1571-1582.

[15] E. Marušić-Paloka and I. Pažanin, On the effects of curved geometry on heat conduction through a distorted pipe. Nonlinear Anal.: Real World App. 11 (2010) 4554-4564.

[16] E. Marušić-Paloka, I. Pažanin and M. Prša, Heat conduction problem in a dilated pipe: existence and uniqueness result. Mediterranean J. Math. 14 (2017) 97.

[17] E. Marušić-Paloka, I. Pažanin and M. Prša, Asymptotic analysis of the heat conduction problem in a dilated pipe. Appl. Math. Comput. 355 (2019) 135-150.

[18] J. Nečas, Les méthodes directes en théorie des équations elliptiques. Masson, Paris (1967).

[19] R. Nittka, Regularity of solutions of linear second order elliptic and parabolic boundary value problems on Lipschitz domains. J. Differ. Equ. 251 (2011) 860-880.

[20] G. Panasenko, Multiscale Modelling of Thin Structures and Composites. Springer (2005).

[21] G. Stampacchia, Equations elliptiques du second ordre à coefficients discontinus. Séminaire Jean Leray 3 (1963-1964) 1-77. 